\title{
Dearomatization and Functionalization of Terpyridine by Lutetium(III) Alkyl Complexes
}

\author{
Kimberly C. Jantunen, ${ }^{\dagger} \S$ Brian L. Scott, ${ }^{\dagger}$ P. Jeffrey Hay, ${ }^{\star}$ John C. Gordon, ${ }^{\dagger}$ \\ and Jaqueline L. Kiplinger*, ${ }^{\dagger}$ \\ Chemistry $^{\dagger}$ and Theoretical ${ }^{+}$Divisions, Los Alamos National Laboratory, Los \\ Alamos, New Mexico, 87545, and ${ }^{\S}$ Department of Chemistry, Simon Fraser \\ University, Burnaby, British Columbia, Canada V5A 1 S6
}

\section{SUPPORTING INFORMATION}

- General procedures and considerations. (S2)

- $\quad$ Synthetic details and characterization data for complexes 3a, 3b, 5a, and 5b. (S3-S6).

- Density functional theory calculation details. (S7-S20).

- $\quad$ Crystallographic details and data for complexes $\mathbf{3 b}$ and 5b. (S21-S31). 


\section{General Procedures and Considerations.}

Methods and Materials. Reactions and manipulations were performed at $21{ }^{\circ} \mathrm{C}$ in a recirculating MBraun 150 B-G nitrogen atmosphere drybox, or using standard Schlenk and high vacuum line techniques. Glassware was dried at $150{ }^{\circ} \mathrm{C}$ before use. All NMR spectra were obtained using a Bruker Avance $300 \mathrm{MHz}$ spectrometer. All NMR spectra were acquired at room temperature $\left(25^{\circ} \mathrm{C}\right)$ in benzene- $d_{6}$. Chemical shifts were referenced to the protio solvent impurity in benzene- $d_{6}$ at $\delta 7.15 \mathrm{ppm}\left({ }^{1} \mathrm{H} \mathrm{NMR}\right)$ and $\delta 128.39 \mathrm{ppm}\left({ }^{13} \mathrm{C} \mathrm{NMR}\right) .{ }^{1} \mathrm{H}$ and ${ }^{13} \mathrm{C}$ NMR assignments were confirmed through the use of DEPT-135 and two-dimensional ${ }^{13} \mathrm{C}\left\{{ }^{1} \mathrm{H}\right\}$ ${ }^{1} \mathrm{H}$ COSY NMR experiments.

Melting points were determined with a Mel-Temp II capillary melting point apparatus equipped with a Fluke 51 II K/J thermocouple using capillary tubes flame-sealed under nitrogen; values are uncorrected. Mass spectrometric (MS) analyses were obtained at the University of California, Berkeley Mass Spectrometry Facility, using a VG ProSpec mass spectrometer. Elemental Analyses were performed at the University of California, Berkeley Microanalytical Facility on a Perkin-Elmer Series II 2400 CHNS analyzer.

Unless otherwise noted, reagents were purchased from commercial suppliers and used without further purification. Celite (Aldrich) and alumina (Brockman I, Aldrich) were dried in vacuo at $250{ }^{\circ} \mathrm{C}$ for $48 \mathrm{~h}$ prior to use. Anhydrous toluene (Aldrich), hexanes (Aldrich), pentane (Aldrich), and tetrahydrofuran (THF) (Aldrich) were passed through a column of activated alumina (A2, $12 \times 32$, Purify) under nitrogen and stored over $4 \AA$ activated molecular sieves prior to use. Benzene- $d_{6}$ (Aldrich) was dried over activated $4 \AA$ molecular sieves prior to use. $\mathrm{Lu}\left(\mathrm{CH}_{2} \mathrm{SiMe}_{3}\right)_{3}(\mathrm{THF})_{2}{ }^{1}$ and $\left(\mathrm{C}_{5} \mathrm{Me}_{5}\right) \mathrm{Lu}\left(\mathrm{CH}_{2} \mathrm{SiMe}_{3}\right)_{2}(\mathrm{THF})^{2}$ were prepared according to the literature procedures. 2,2':6',2'’terpyridine (tpy) (Aldrich) and 4,4',4'’Tri-tert-Butyl2,2':6',2'’terpyridine ( ${ }^{\mathrm{t}} \mathrm{Bu}_{3}$ tpy) (Aldrich) were purified by recrystallization from toluene at -35 ${ }^{\circ} \mathrm{C}$. X-ray data were collected and the structures solved at the Los Alamos National Laboratory X-ray facility. 


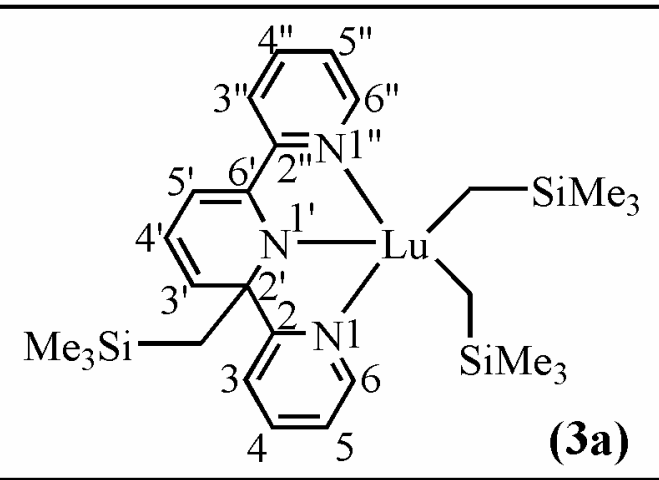

Synthesis of 3a. A $125 \mathrm{~mL}$ side-arm flask equipped with a magnetic stir bar was charged with $\mathrm{Lu}\left(\mathrm{CH}_{2} \mathrm{SiMe}_{3}\right)_{3}(\mathrm{THF})_{2}(\mathbf{1})(0.994 \mathrm{~g}, 1.71 \mathrm{mmol})$ and toluene $(40 \mathrm{~mL})$. To the resulting clear, colorless solution a $20 \mathrm{~mL}$ toluene solution of 2,2':6',2', terpyridine (2a) (0.399 g, $1.71 \mathrm{mmol})$ was added portion-wise with stirring. The resultant reaction mixture immediately turned very dark greenish-orange in color and was stirred at room temperature for $15 \mathrm{~h}$. The volatiles were removed under reduced pressure to give 3a as an analytically pure dark greenish-orange powder (1.088 g, 1.62 mmol, 95 \%). ${ }^{1} \mathrm{H}$ NMR: $\delta 8.51$ (d, 1H, $5.2 \mathrm{~Hz}, H 6$ or $\left.H 6^{\prime \prime}\right), 8.39$ (d, 1H, $5.2 \mathrm{~Hz}$, $H 6$ or $\left.H 6^{\prime \prime}\right), 7.33$ (d, 1H, $8.2 \mathrm{~Hz}, H 3$ or $\left.H 3^{\prime \prime}\right), 6.96$ (m, 2H, $H 3$ or $H 3^{\prime \prime}$ and $H 4$ or $\left.H 4^{\prime \prime}\right), 6.84$ (m, $1 \mathrm{H}, H 4$ or $\left.H 4^{\prime \prime}\right), 6.52$ (m, $1 \mathrm{H}, H 5$ or $\left.H 5^{\prime \prime}\right), 6.43$ (m, $1 \mathrm{H}, H 5$ or $\left.H 5^{\prime \prime}\right), 6.34(\mathrm{dd}, 1 \mathrm{H}, 8.5 \mathrm{~Hz}, 6.0$ Hz, $\left.H 4^{\prime}\right), 5.75$ (d, 1H, $6.0 \mathrm{~Hz}, H 3^{\prime}$ or $\left.H 5^{\prime}\right), 5.20$ (d, 1H, $8.5 \mathrm{~Hz}, H 3^{\prime}$ or $\left.H 5^{\prime}\right), 2.09$ (d, $1 \mathrm{H}, 14.6$ $\mathrm{Hz}, \mathrm{CH}_{2} \mathrm{SiMe}_{3}$ ), 0.99 (d, 1H, $14.6 \mathrm{~Hz}, \mathrm{CH}_{2} \mathrm{SiMe}_{3}$ ), 0.48 (s, 9H, $\mathrm{CH}_{2} \mathrm{SiMe}_{3}$ ), -0.07 (s, 9H, $\mathrm{CH}_{2} \mathrm{SiMe}$ ), $-0.29\left(\mathrm{~s}, 9 \mathrm{H}, \mathrm{CH}_{2} \mathrm{SiMe}\right.$ ), $-0.34\left(\mathrm{~s}, 2 \mathrm{H}, \mathrm{CH}_{2} \mathrm{SiMe}_{3}\right)$. The remaining $\mathrm{CH}_{2} \mathrm{SiMe}_{3}$ resonance is obscured by the resonance at $\delta-0.29 .{ }^{13} \mathrm{C}\left\{{ }^{1} \mathrm{H}\right\} \mathrm{NMR}: \delta 173.75$ (s, $C 2, C 6{ }^{\prime}$ or $\left.C 2^{\prime \prime}\right)$, $162.30\left(\mathrm{~s}, C 2, C 6^{\prime}\right.$ or $\left.C 2^{\prime \prime}\right), 148.82\left(\mathrm{~s}, C 2, C 6^{\prime}\right.$ or $\left.C 2^{\prime \prime}\right), 145.85$ (s, $C 6$ or $\left.C 6^{\prime \prime}\right), 145.53$ (s, $C 6$ or $\left.C 6^{\prime \prime}\right), 140.23$ (s, C3,C3", $C 4$ or $\left.C 4^{\prime \prime}\right), 138.93$ (s, $C 4$ or $\left.C 4^{\prime \prime}\right), 125.27\left(\mathrm{~s}, C 4^{\prime}\right), 123.32$ (s, C3, $C 3^{\prime \prime}, C 4$ or $\left.C 4^{\prime \prime}\right), 122.39$ (s, $C 5$ or $\left.C 5^{\prime \prime}\right), 122.05$ (s, $C 5$ or $\left.C 5^{\prime \prime}\right), 121.33$ (s, $C 3$ or $\left.C 3^{\prime \prime}\right), 119.40$ (s, $C 3^{\prime}$ or $\left.C 5^{\prime}\right), 98.10\left(\mathrm{~s}, C 3^{\prime}\right.$ or $\left.C 5^{\prime}\right), 68.43\left(\mathrm{~s}, C 2^{\prime}\right), 45.65\left(\mathrm{~s}, C \mathrm{H}_{2} \mathrm{SiMe}_{3}\right), 44.51\left(\mathrm{~s}, \mathrm{CH}_{2} \mathrm{SiMe}_{3}\right)$, 32.84 (s, $\left.\mathrm{CH}_{2} \mathrm{SiMe}_{3}\right), 5.20$ (s, $\mathrm{CH}_{2} \mathrm{SiMe}_{3}$ ), 4.08 (s, $\left.\mathrm{CH}_{2} \mathrm{SiMe}_{3}\right), 0.50$ (s, $\mathrm{CH}_{2} \mathrm{SiMe}_{3}$ ). Anal. Calcd. for $\mathrm{C}_{27} \mathrm{H}_{44} \mathrm{~N}_{3} \mathrm{LuSi}_{3}(669.89 \mathrm{~g} / \mathrm{mol})$ : C, 48.71; H, 6.62; N, 6.27. Found: C, 48.33; N, 6.47; H, 6.12. $\mathrm{MS}(\mathrm{EI}, 70 \mathrm{eV}): m / z 670\left(\mathrm{M}^{+}\right), 582\left(\mathrm{M}^{+}-\mathrm{CH}_{2} \mathrm{SiMe}_{3}\right), 494\left(\mathrm{M}^{+}-2 \mathrm{CH}_{2} \mathrm{SiMe}_{3}\right) . \mathrm{Mp}=85-86$ ${ }^{\circ} \mathrm{C}$. 


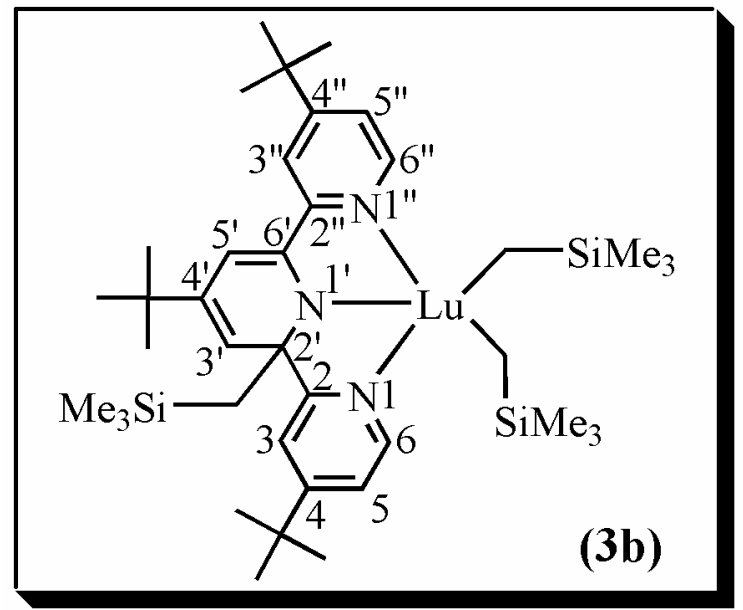

Synthesis of 3b. A $125 \mathrm{~mL}$ side-arm flask equipped with a stir bar was charged with $\mathrm{Lu}\left(\mathrm{CH}_{2} \mathrm{SiMe}_{3}\right)_{3}(\mathrm{THF})_{2}(\mathbf{1})(0.808 \mathrm{~g}, 1.39 \mathrm{mmol})$ and toluene $(40 \mathrm{~mL})$. To the resulting clear, colorless solution a $20 \mathrm{~mL}$ toluene solution of 4,4',4', Tri-tert-Butyl-2,2':6',2',terpyridine (2b) (0.559 g, $1.39 \mathrm{mmol})$ was added portion-wise with stirring. The resultant reaction mixture immediately turned very dark greenish-orange in color and was stirred at room temperature for $15 \mathrm{~h}$. The volatiles were removed under reduced pressure to give $\mathbf{3 b}$ as an analytically pure dark greenish-orange powder (1.046 g, $1.25 \mathrm{mmol}, 90 \%)$. ${ }^{1} \mathrm{H} \mathrm{NMR}: \delta 8.56$ (d, 1H, $5.8 \mathrm{~Hz}, H 6$ or $\left.H 6^{\prime \prime}\right), 8.48$ (d, 1H, $5.8 \mathrm{~Hz}, H 6$ or $\left.H 6^{\prime \prime}\right), 7.93$ (s, 1H, $H 3$ or $\left.H 3^{\prime \prime}\right), 7.60$ (s, 1H, H3 or $\left.H 3^{\prime \prime}\right), 6.76$ (dd, 1H, $5.8 \mathrm{~Hz}, 1.6 \mathrm{~Hz}, H 5$ or $\left.H 5^{\prime \prime}\right), 6.70(\mathrm{dd}, 1 \mathrm{H}, 5.8 \mathrm{~Hz}, 1.6 \mathrm{~Hz}, H 5$ or H5'), 6.17 (s, 1H, H3' or $\left.H 5^{\prime}\right), 5.27$ (s, 1H, H3' or $\left.H 5^{\prime}\right), 2.31$ (d, 1H, $\left.14.5 \mathrm{~Hz}, \mathrm{CH}_{2} \mathrm{SiMe}_{3}\right), 1.33$ (s, 9H, CMe 3 ), 1.18 (d, $1 \mathrm{H}, 14.5 \mathrm{~Hz}, \mathrm{CH}_{2} \mathrm{SiMe}_{3}$ ), 0.98 (s, 9H, CMe $), 0.95$ (s, 9H, CMe $), 0.56$ (s, 9H, $\left.\mathrm{CH}_{2} \mathrm{SiMe}_{3}\right), 0.22$ (d, 2H, $6.3 \mathrm{~Hz}, \mathrm{CH}_{2} \mathrm{SiMe}_{3}$ ), -0.05 (s, 9H, $\mathrm{CH}_{2} \mathrm{SiMe}_{3}$ ), -0.28 (s, 9H, $\mathrm{CH}_{2} \mathrm{Si}_{2} \mathrm{e}_{3}$ ). The remaining $\mathrm{CH}_{2} \mathrm{SiMe}_{3}$ resonance is obscured by the resonance at $\delta-0.28 .{ }^{13} \mathrm{C}\left\{{ }^{1} \mathrm{H}\right\} \mathrm{NMR}: \delta 174.40$ (s, quat aryl $C$ ), 164.97 (s, quat aryl $C$ ), 163.73 (s, quat aryl $C$ ), 162.91 (s, quat aryl $C$ ), 149.71 (s, quat aryl C), 146.17 (s, quat aryl C), 145.90 (s, $C 6$ or $\left.C 6^{\prime \prime}\right), 145.69$ (s, $C 6$ or $\left.C 6^{\prime \prime}\right), 120.27$ (s, $C 3$ or $\left.C 3^{\prime \prime}\right), 120.05$ (s, $C 5$ or $\left.C 5^{\prime \prime}\right), 119.91$ (s, $C 5$ or $\left.C 5^{\prime \prime}\right), 117.51$ (s, $C 3$ or $\left.C 3^{\prime \prime}\right), 113.22$ (s, $C 3^{\prime}$ or $\left.C 5^{\prime}\right), 96.92$ (s, $C 3^{\prime}$ or $\left.C 5^{\prime}\right), 69.50\left(\mathrm{~s}, C 2^{\prime}\right), 45.46\left(\mathrm{~s}, \mathrm{CH}_{2} \mathrm{SiMe}_{3}\right), 43.89\left(\mathrm{~s}, \mathrm{CH}_{2} \mathrm{SiMe}_{3}\right), 35.54$ (s, $C \mathrm{Me}_{3}$ ), 35.43 (s, $C \mathrm{Me}_{3}$ ), 34.30 (s, $C \mathrm{Me}_{3}$ ), 32.01 (s, $\mathrm{CH}_{2} \mathrm{SiMe}_{3}$ ), 30.57 (s, $\mathrm{CMe}_{3}$ ), 30.40 (s, $\mathrm{CMe}_{3}$ ), 30.33 (s, $\mathrm{CMe}$ ), 5.32 (s, $\left.\mathrm{CH}_{2} \mathrm{SiMe}_{3}\right), 4.24$ (s, $\mathrm{CH}_{2} \mathrm{SiMe}_{3}$ ), 0.61 (s, $\left.\mathrm{CH}_{2} \mathrm{SiMe}_{3}\right)$. Anal. Calcd. for $\mathrm{C}_{39} \mathrm{H}_{68} \mathrm{~N}_{3} \mathrm{LuSi}_{3}$ (838.21 g/mol): C, 55.88; H, 8.18; N, 5.01. Found: C, 55.84; N, 8.41; $\mathrm{H}, 4.95 . \mathrm{Mp}=164-166^{\circ} \mathrm{C}$. 


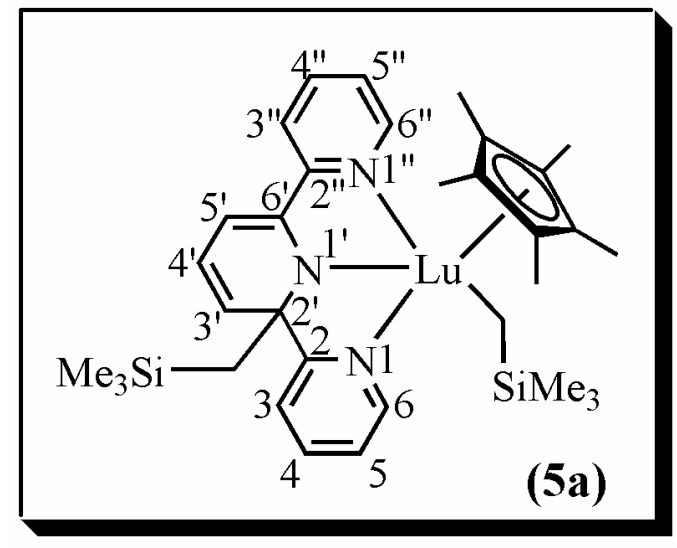

Synthesis of 5a. A $125 \mathrm{~mL}$ side-arm flask equipped with a stir bar was charged with $\left(\mathrm{C}_{5} \mathrm{Me}_{5}\right) \mathrm{Lu}\left(\mathrm{CH}_{2} \mathrm{SiMe}_{3}\right)_{2}(\mathrm{THF})(4)(501 \mathrm{mg}, 0.90 \mathrm{mmol})$ and hexanes $(25 \mathrm{~mL})$. To the resulting clear, colorless solution a $20 \mathrm{~mL}$ hexanes solution of $2,2^{\prime}: 6^{\prime}, 2^{\prime}$ 'terpyridine (2a) $(0.210 \mathrm{~g}, 0.90$ mmol) was added portion-wise with stirring. The resultant reaction mixture immediately turned very dark orange-brown in color and was stirred at room temperature for $1 \mathrm{~h}$. The volatiles were removed under reduced pressure to give 5a a dark greenish-orange powder $(0.614 \mathrm{~g}, 95 \%)$. The product was recrystallized from pentane at $-30{ }^{\circ} \mathrm{C}$. ${ }^{1} \mathrm{H}$ NMR: $\delta 8.33\left(\mathrm{~d}, 1 \mathrm{H}, 5.2 \mathrm{~Hz}, H 6\right.$ or $\left.H 6^{\prime \prime}\right)$, 8.22 (d, 1H, $5.2 \mathrm{~Hz}, H 6$ or $\left.H 6^{\prime \prime}\right), 7.29$ (d, 1H, 8.0 Hz, H3 or H3"), 7.02 (m, 2H, H3 or H3" and $H 4$ or $\left.H 4^{\prime \prime}\right), 6.88$ (m, 1H, $H 4$ or $\left.H 4^{\prime \prime}\right), 6.57$ (m, $1 \mathrm{H}, H 5$ or $\left.H 5^{\prime \prime}\right), 6.47$ (m, 1H, H5 or $\left.H 5^{\prime \prime}\right), 6.35$ (dd, 1H, 8.2 Hz, $\left.5.8 \mathrm{~Hz}, H 4^{\prime}\right), 5.83$ (d, 1H, $5.8 \mathrm{~Hz}, H 3^{\prime}$ or $\left.H 5^{\prime}\right), 5.17$ (d, 1H, $8.2 \mathrm{~Hz}, H 3^{\prime}$ or $H 5^{\prime}$ ), 2.03 (d, 1H, $\left.14.5 \mathrm{~Hz}, \mathrm{CH}_{2} \mathrm{SiMe}_{3}\right), 0.96$ (d, 1H, $\left.14.5 \mathrm{~Hz}, \mathrm{CH}_{2} \mathrm{SiMe}_{3}\right), 1.82$ (s, 15H, $\left.\mathrm{C}_{5} \mathrm{Me}_{5}\right), 0.30$ (s, 9H, $\mathrm{CH}_{2} \mathrm{SiMe}_{3}$ ), -0.33 (s, 9H, $\mathrm{CH}_{2} \mathrm{SiMe}$ ), -0.57 (d, 1H, $\left.10.7 \mathrm{~Hz}, \mathrm{CH}_{2} \mathrm{SiMe}_{3}\right),-0.72$ (d, 1H, $\left.10.7 \mathrm{~Hz}, \mathrm{CH}_{2} \mathrm{SiMe}_{3}\right) .{ }^{13} \mathrm{C}\left\{{ }^{1} \mathrm{H}\right\}$ NMR: $\delta 172.94$ (s, C2,C6' or $\left.C 2^{\prime \prime}\right), 162.39$ (s, C2, C6' or $\left.C 2^{\prime \prime}\right)$, 149.13 (s, $C 2, C 6^{\prime}$ or $\left.C 2^{\prime \prime}\right), 147.90$ (s, $C 6$ or $\left.C 6^{\prime \prime}\right), 147.75$ (s, $C 6$ or $\left.C 6^{\prime \prime}\right), 139.07$ (s, $C 3, C 3^{\prime \prime}, C 4$ or $\left.C 4^{\prime \prime}\right), 138.05$ (s, $C 4$ or $\left.C 4^{\prime \prime}\right), 125.61\left(\mathrm{~s}, C 4^{\prime}\right), 123.39$ (s, $C 3, C 3^{\prime \prime}, C 4$ or $\left.C 4^{\prime \prime}\right), 121.53$ (s, $C 5$ or $\left.C 5^{\prime \prime}\right), 121.38\left(\mathrm{~s}, C 5\right.$ or $\left.C 5^{\prime \prime}\right), 120.50\left(\mathrm{~s}, C 3\right.$ or $\left.C 3^{\prime \prime}\right), 117.75\left(\mathrm{~s}, C 3^{\prime}\right.$ or $\left.C 5^{\prime}\right), 116.73\left(\mathrm{~s}, C_{5} \mathrm{Me}_{5}\right)$, $98.35\left(\mathrm{~s}, C 3^{\prime}\right.$ or $\left.C 5^{\prime}\right), 67.67\left(\mathrm{~s}, C 2^{\prime}\right), 34.77\left(\mathrm{~s}, \mathrm{CH}_{2} \mathrm{SiMe}_{3}\right), 30.50\left(\mathrm{~s}, \mathrm{CH}_{2} \mathrm{SiMe}_{3}\right), 11.60$ (s, $\left.\mathrm{C}_{5} \mathrm{Me}_{5}\right), 5.48\left(\mathrm{~s}, \mathrm{CH}_{2} \mathrm{SiMe}\right.$ ), $0.61\left(\mathrm{~s}, \mathrm{CH}_{2} \mathrm{SiMe}\right.$ ). Anal. Calcd. for $\mathrm{C}_{33} \mathrm{H}_{48} \mathrm{~N}_{3} \mathrm{LuSi}_{2} \cdot \mathrm{C}_{6} \mathrm{H}_{12}(717.28$ g/mol): C, 57.77; H, 7.65 N, 5.32. Found: C, 57.52; N, 7.20; H, 4.98. MS(EI, 70 eV): m/z 717 $\left(\mathrm{M}^{+}\right), 630\left(\mathrm{M}^{+}-\mathrm{CH}_{2} \mathrm{SiMe}_{3}\right) . \mathrm{Mp}=99-100^{\circ} \mathrm{C}$. 


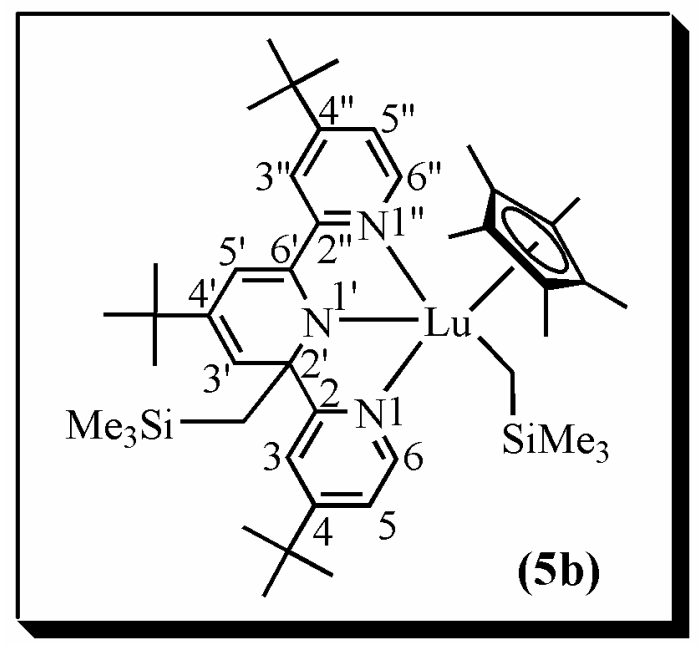

Synthesis of 5b. A $125 \mathrm{~mL}$ side-arm flask equipped with a stir bar was charged with $\left(\mathrm{C}_{5} \mathrm{Me}_{5}\right) \mathrm{Lu}\left(\mathrm{CH}_{2} \mathrm{SiMe}_{3}\right)_{2}$ (THF) (4) (0.249 g, $\left.0.45 \mathrm{mmol}\right)$ and hexanes $(25 \mathrm{~mL})$. To the resulting clear, colorless solution a $20 \mathrm{~mL}$ hexanes solution of 4,4',4' 'Tri-tert-Butyl-2,2':6',2' 'terpyridine (2b) $(0.180 \mathrm{~g}, 0.45 \mathrm{mmol})$ was added portion-wise with stirring. The resultant reaction mixture immediately turned very dark green-orange in color and was stirred at room temperature for $1 \mathrm{~h}$. The volatiles were removed under reduced pressure to give $\mathbf{5 b}$ as an analytically pure dark greenish-orange powder $(0.380 \mathrm{~g}, 0.43 \mathrm{mmol}, 96 \%) .{ }^{1} \mathrm{H} \mathrm{NMR}: \delta 8.38$ (d, 1H, $5.8 \mathrm{~Hz}, H 6$ or $\left.H 6^{\prime \prime}\right), 8.30$ (d, 1H, $5.8 \mathrm{~Hz}, H 6$ or $\left.H 6^{\prime \prime}\right), 7.87$ (d, 1H, $1.6 \mathrm{~Hz}, H 3$ or $\left.H 3^{\prime \prime}\right), 7.59$ (d, 1H, $1.6 \mathrm{~Hz}, H 3$ or $\left.H 3^{\prime \prime}\right), 6.84\left(\mathrm{dd}, 1 \mathrm{H}, 5.8 \mathrm{~Hz}, 1.9 \mathrm{~Hz}, H 5\right.$ or $\left.H 5^{\prime \prime}\right), 6.76$ (dd, 1H, $5.8 \mathrm{~Hz}, 1.9 \mathrm{~Hz}, H 5$ or $\left.H 5^{\prime \prime}\right)$, $6.25\left(\mathrm{~d}, 1 \mathrm{H}, 1.4 \mathrm{~Hz}, H 3^{\prime}\right.$ or $\left.H 5^{\prime}\right), 5.26\left(\mathrm{~d}, 1 \mathrm{H}, 1.4 \mathrm{~Hz}, H 3^{\prime}\right.$ or $\left.H 5^{\prime}\right), 2.17(\mathrm{~d}, 1 \mathrm{H}, 14.4 \mathrm{~Hz}$, $\mathrm{CH}_{2} \mathrm{SiMe}_{3}$ ), 1.91 (s, 15H, C $\mathrm{Me}_{5}$ ), 1.38 (s, 9H, CMe $), 1.23$ (d, 1H, $14.4 \mathrm{~Hz}, \mathrm{CH}_{2} \mathrm{SiMe}_{3}$ ), 1.03 (s, 9H, $\mathrm{CMe}_{3}$ ), 0.99 (s, 9H, CMe $), 0.37$ (s, 9H, $\mathrm{CH}_{2} \mathrm{SiMe}$ ), -0.30 (s, 9H, $\mathrm{CH}_{2} \mathrm{SiMe}_{3}$ ), -0.51 (d, 1H, $10.7 \mathrm{~Hz}, \mathrm{CH}_{2} \mathrm{SiMe}_{3}$ ), -0.68 (d, $1 \mathrm{H}, 10.7 \mathrm{~Hz}, \mathrm{CH}_{2} \mathrm{SiMe}_{3}$ ). ${ }^{13} \mathrm{C}\left\{{ }^{1} \mathrm{H}\right\} \mathrm{NMR}: \delta 173.56$ (s, quat aryl C), 163.71 (s, quat aryl C), 162.81 (s, quat aryl C), 162.75 (s, quat aryl C), 149.97 (s, quat aryl C), 148.01 (s, $C 6$ or $\left.C 6^{\prime \prime}\right), 147.88$ (s, C6 or $\left.C 6^{\prime \prime}\right), 146.34$ (s, aryl C), 120.05 (s, C3 or $\left.C 3^{\prime \prime}\right)$, $119.27\left(\mathrm{~s}, C 5\right.$ or $\left.C 5^{\prime \prime}\right), 119.08\left(\mathrm{~s}, C 5\right.$ or $\left.C 5^{\prime \prime}\right), 116.79\left(\mathrm{~s}, C 3\right.$ or $\left.C 3^{\prime \prime}\right), 116.57\left(\mathrm{~s}, C \mathrm{Me}_{5}\right), 111.51$ (s, $C 3^{\prime}$ or $\left.C 5^{\prime}\right), 96.78\left(\mathrm{~s}, C 3^{\prime}\right.$ or $\left.C 5^{\prime}\right), 68.81\left(\mathrm{~s}, C 2^{\prime}\right), 35.43\left(\mathrm{~s}, C \mathrm{Me}_{3}\right), 35.30\left(\mathrm{~s}, C \mathrm{Me}_{3}\right), 34.31$ (s, $C \mathrm{Me}_{3}$ ), 34.28 (s, $\mathrm{CH}_{2} \mathrm{SiMe}_{3}$ ), 31.04 (s, $\mathrm{CH}_{2} \mathrm{SiMe}_{3}$ ), 30.74 (s, $\mathrm{CMe}$ ), 30.52 (s, $\mathrm{CMe}_{3}$ ), 30.44 (s, $\left.\mathrm{CMe}_{3}\right), 11.79\left(\mathrm{~s}, \mathrm{CMe}_{5}\right) 5.61\left(\mathrm{~s}, \mathrm{CH}_{2} \mathrm{SiMe} e_{3}\right), 0.70$ (s, $\left.\left.\mathrm{CH}_{2} \mathrm{SiMe}\right)_{3}\right)$ Anal. Calcd. for $\mathrm{C}_{45} \mathrm{H}_{72} \mathrm{~N}_{3} \mathrm{LuSi}_{2}$ (886.23 g/mol): C, 60.99; H, 8.19; N, 4.74. Found: C, 60.61; N, 8.04; H, 4.66. $\mathrm{MS}(\mathrm{EI}, 70 \mathrm{eV}): m / z 886\left(\mathrm{M}^{+}\right), 798\left(\mathrm{M}^{+}-\mathrm{CH}_{2} \mathrm{SiMe}_{3}\right) . \mathrm{Mp}=160-161{ }^{\circ} \mathrm{C}$. 


\section{Description of DFT calculations}

Calculations on the Lu complexes used a "small core" effective core potential on Lu that replaced 28 core electrons and employed a $10 \mathrm{~s} 8 \mathrm{p} 5 \mathrm{~d} 4 \mathrm{f} \mathrm{basis}^{3}$. A 6-31g basis was used for the ligands. Optimizations were carried out using no symmetry constraints using the B3LYP functional. The relative energies of the $\mathrm{Cp}^{*}$-Lu complexes were checked using 6-31 $\mathrm{g}^{*}$ basis on the ligands, which showed very little change from the results with 6-31g basis. All calculations used Gaussian 03, Revision C.02, M. J. Frisch et al., Gaussian, Inc., Wallingford, CT, 2004.

Table S1. Calculated total energies for lutetium(III) complexes.

\begin{tabular}{lll}
\hline Structure & Symbol & Total energy (a.u.) \\
\hline$\left(\mathrm{C}_{5} \mathrm{Me}_{5}\right) \mathrm{Lu}(\mathrm{R}$-tpy)(R $)$ & $\mathbf{5 a}$ & -3265.75376 \\
$\left(\mathrm{C}_{5} \mathrm{Me}_{5}\right) \mathrm{Lu}(\mathrm{tpy})(\mathrm{R})_{2}$ & $\mathbf{6}$ & -3265.71636 \\
$\left(\mathrm{C}_{5} \mathrm{Me}_{5}\right) \mathrm{Lu}(\mathrm{tpy})\left(\mathrm{CH}-\mathrm{SiMe}_{3}\right)$ & $\mathbf{8}$ & -2816.59133 \\
$\mathrm{Lu}(\mathrm{R}-\mathrm{tpy})(\mathrm{R})_{2}$ & $\mathbf{3 a}$ & -3324.23264 \\
$\mathrm{Lu}(\mathrm{tpy})(\mathrm{R})_{3}$ & 7 & -3324.20959 \\
$\mathrm{Lu}\left(\mathrm{tpy}_{2}\right)(\mathrm{R})\left(\mathrm{CH}-\mathrm{SiMe}_{3}\right)$ & $\mathbf{9}$ & -2875.06621 \\
$\mathrm{SiMe}_{4}$ & & -449.12927 \\
\hline
\end{tabular}

Table S2. Cartesian coordinates for 5a.

$\begin{array}{llll}71 & -0.644128 & -0.402741 & -0.138473 \\ 14 & -2.087532 & 2.712245 & -2.062754 \\ 7 & 1.250689 & -1.229827 & -1.434201 \\ 7 & 1.299060 & -0.250720 & 1.024555 \\ 7 & 1.046430 & 1.786933 \\ 6 & -0.906659 & -1.346232 & -0.819825 \\ 6 & 2.459044 & 0.041179 & 2.368204 \\ 6 & 1.205673 & 0.925725 & 2.726589 \\ 6 & 0.082491 & 2.492820 & 4.165899 \\ 6 & -1.073717 & -0.804377 & 0.594842 \\ 6 & 2.606415 & -0.557806 & 3.328083 \\ 6 & 2.007895 & -1.924792 & -1.496354 \\ 6 & 3.545594 & -1.672510 & 2.904844 \\ 6 & 2.812902 & 1.645341 & 3.932038\end{array}$




\begin{tabular}{|c|c|c|c|}
\hline 6 & 3.399354 & -2.353855 & -2.814731 \\
\hline 6 & 3.024255 & -1.901507 & 1.581773 \\
\hline 6 & 2.165383 & -2.182547 & -3.453922 \\
\hline 6 & 1.120090 & -1.619537 & -2.729922 \\
\hline 6 & -2.074985 & 2.617231 & 3.192434 \\
\hline 6 & -1.245111 & -2.936853 & 0.516693 \\
\hline 6 & -1.953936 & 1.873919 & 2.021464 \\
\hline 6 & -2.006528 & -2.127429 & 1.416017 \\
\hline 6 & 1.090235 & 1.099549 & -1.927372 \\
\hline 1 & -0.065898 & 1.362839 & -2.258825 \\
\hline 1 & -1.493351 & 0.416787 & -2.703441 \\
\hline 6 & -2.879610 & -1.838743 & -0.716722 \\
\hline 6 & -3.016764 & -1.445692 & 0.653532 \\
\hline 6 & -1.784270 & -2.759652 & -0.803539 \\
\hline 6 & -1.446390 & -3.601880 & -2.006428 \\
\hline 1 & 0.388562 & -3.885690 & -2.035788 \\
\hline 1 & -1.680038 & -3.092457 & -2.949022 \\
\hline 1 & -2.026117 & -4.537184 & -2.003866 \\
\hline 6 & 3.826961 & -1.485369 & -1.832383 \\
\hline 1 & -3.337848 & -1.519728 & -2.812606 \\
\hline 1 & -4.239311 & -0.478694 & -1.713612 \\
\hline 1 & -4.677971 & -2.182299 & -1.873612 \\
\hline 6 & -1.868860 & -2.117045 & 2.916878 \\
\hline 1 & -2.272965 & -1.198798 & 3.357545 \\
\hline 1 & -0.824165 & -2.201544 & 3.237972 \\
\hline 1 & -2.414389 & -2.956995 & 3.372668 \\
\hline 6 & -0.177516 & -3.924795 & 0.908780 \\
\hline 1 & 0.516153 & -4.125988 & 0.084144 \\
\hline 1 & -0.615712 & -4.891475 & 1.200852 \\
\hline 1 & 0.422202 & -3.567828 & 1.752481 \\
\hline 6 & -4.167051 & -0.657677 & 1.223867 \\
\hline 1 & -5.018555 & -1.315397 & 1.455045 \\
\hline 1 & -4.537658 & 0.101063 & 0.524557 \\
\hline 1 & -3.900702 & -0.150506 & 2.157682 \\
\hline 14 & 3.584063 & 1.969937 & -0.373246 \\
\hline 6 & 3.729458 & 0.304236 & 0.609928 \\
\hline 1 & 4.680994 & -0.164141 & 0.319727 \\
\hline
\end{tabular}




\begin{tabular}{|c|c|c|c|}
\hline 1 & 3.853907 & 0.594978 & 1.661665 \\
\hline 6 & -2.109928 & 3.402601 & -3.867871 \\
\hline 1 & -1.090314 & 3.606148 & -4.217517 \\
\hline 1 & -2.683901 & 4.336001 & -3.930408 \\
\hline 1 & -2.562684 & 2.677502 & -4.555524 \\
\hline 6 & 3.191487 & 1.756715 & -2.239940 \\
\hline 1 & 2.181306 & 1.369403 & -2.401193 \\
\hline 1 & 3.903028 & 1.075296 & -2.719595 \\
\hline 1 & 3.263662 & 2.729339 & -2.741441 \\
\hline 6 & 2.323091 & 3.166494 & 0.433040 \\
\hline 1 & 2.379572 & 4.155046 & -0.038282 \\
\hline 1 & 2.535149 & 3.287796 & 1.501810 \\
\hline 1 & 1.300405 & 2.793635 & 0.329935 \\
\hline 6 & -1.342193 & 4.117172 & -0.973710 \\
\hline 6 & -3.944218 & 2.521277 & -1.572338 \\
\hline 6 & 5.332279 & 2.764948 & -0.232710 \\
\hline 1 & 5.350644 & 3.749701 & -0.714642 \\
\hline 1 & 6.091352 & 2.137705 & -0.715563 \\
\hline 1 & 5.620571 & 2.897889 & 0.816854 \\
\hline 1 & 0.802119 & 1.555282 & 4.660434 \\
\hline 1 & 1.888625 & -0.340010 & 4.381631 \\
\hline 1 & 4.497558 & -2.026082 & -0.990087 \\
\hline 1 & 2.015828 & -2.480213 & -4.484663 \\
\hline 1 & -2.698013 & 1.933643 & 1.238176 \\
\hline 1 & 0.146834 & -1.463045 & -3.175663 \\
\hline 1 & 3.516278 & -2.798756 & 1.219834 \\
\hline 1 & -2.922645 & 3.276318 & 3.333795 \\
\hline 1 & -4.073762 & 2.149416 & -0.549237 \\
\hline 1 & -4.451949 & 3.490984 & -1.646540 \\
\hline 1 & -4.453813 & 1.824289 & -2.248040 \\
\hline 1 & -0.296438 & 4.302936 & -1.245364 \\
\hline 1 & -1.901362 & 5.048750 & -1.125752 \\
\hline 1 & -1.370085 & 3.873531 & 0.093771 \\
\hline 1 & -1.130422 & 3.059923 & 5.088911 \\
\hline 1 & 4.235809 & -2.802967 & -3.339681 \\
\hline 1 & 3.184080 & -2.368990 & 3.652212 \\
\hline
\end{tabular}




\begin{tabular}{|c|c|c|c|}
\hline 71 & -0.419154 & -0.401838 & -0.065124 \\
\hline 14 & -3.604822 & 1.343630 & -1.623861 \\
\hline 6 & -0.074295 & -2.842572 & 1.191863 \\
\hline 6 & -0.602778 & -1.984085 & 2.208855 \\
\hline 6 & -2.228508 & 0.026459 & -1.631617 \\
\hline 1 & -1.655012 & 0.232781 & -2.556553 \\
\hline 1 & -2.737579 & -0.940684 & -1.788405 \\
\hline 6 & -2.290934 & -2.401940 & 0.677512 \\
\hline 6 & -1.971120 & -1.701807 & 1.881694 \\
\hline 6 & -1.118462 & -3.090242 & 0.238533 \\
\hline 6 & -1.061656 & -4.086886 & -0.889247 \\
\hline 1 & -0.043827 & -4.217069 & -1.274017 \\
\hline 1 & -1.697822 & -3.792279 & -1.733192 \\
\hline 1 & -1.407302 & -5.080851 & -0.566015 \\
\hline 6 & -3.668249 & -2.562555 & 0.096628 \\
\hline 1 & -3.638086 & -2.818486 & -0.967747 \\
\hline 1 & -4.264586 & -1.651126 & 0.192914 \\
\hline 1 & -4.218841 & -3.369038 & 0.605205 \\
\hline 6 & 0.062364 & -1.666718 & 3.521105 \\
\hline 1 & -0.310159 & -0.733518 & 3.958550 \\
\hline 1 & 1.150944 & -1.576696 & 3.428154 \\
\hline 1 & -0.126071 & -2.459155 & 4.261997 \\
\hline 6 & 1.238771 & -3.574913 & 1.260968 \\
\hline 1 & 1.648256 & -3.794148 & 0.267810 \\
\hline 1 & 1.126342 & -4.542050 & 1.775046 \\
\hline 1 & 1.999040 & -3.011042 & 1.813074 \\
\hline 6 & -2.960292 & -1.006821 & 2.780449 \\
\hline 1 & -3.401867 & -1.706502 & 3.507026 \\
\hline 1 & -3.792109 & -0.571767 & 2.215707 \\
\hline 1 & -2.498269 & -0.201279 & 3.363751 \\
\hline 14 & 1.944829 & 2.813247 & -1.560082 \\
\hline 6 & 0.478770 & 1.631719 & -1.391822 \\
\hline 1 & -0.425141 & 2.216516 & -1.169614 \\
\hline 1 & 0.318202 & 1.156754 & -2.372294 \\
\hline 6 & -4.694658 & 1.267323 & -3.219932 \\
\hline
\end{tabular}




\begin{tabular}{|c|c|c|c|}
\hline 1 & -4.082233 & 1.448727 & -4.111772 \\
\hline 1 & -5.492983 & 2.020253 & -3.194569 \\
\hline 1 & -5.159175 & 0.279138 & -3.325655 \\
\hline 6 & 2.393287 & 3.782409 & 0.050216 \\
\hline 1 & 2.851486 & 3.132212 & 0.802982 \\
\hline 1 & 1.504323 & 4.248237 & 0.492134 \\
\hline 1 & 3.108274 & 4.579770 & -0.187943 \\
\hline 6 & 3.565085 & 1.949204 & -2.161647 \\
\hline 1 & 4.302903 & 2.705669 & -2.456627 \\
\hline 1 & 3.369355 & 1.311709 & -3.032450 \\
\hline 1 & 4.012686 & 1.329802 & -1.376997 \\
\hline 6 & -2.957717 & 3.160843 & -1.535338 \\
\hline 6 & -4.854857 & 1.174044 & -0.158260 \\
\hline 6 & 1.578775 & 4.177456 & -2.883711 \\
\hline 1 & 2.428808 & 4.864003 & -2.991928 \\
\hline 1 & 0.696151 & 4.764010 & -2.601147 \\
\hline 1 & 1.378502 & 3.722045 & -3.861282 \\
\hline 1 & -4.363946 & 1.217196 & 0.821556 \\
\hline 1 & -5.586195 & 1.990986 & -0.204039 \\
\hline 1 & -5.406957 & 0.228826 & -0.213489 \\
\hline 1 & -2.247637 & 3.369557 & -2.343136 \\
\hline 1 & -3.802605 & 3.854362 & -1.634173 \\
\hline 1 & -2.452253 & 3.377755 & -0.586769 \\
\hline 6 & 1.017225 & 2.354180 & 3.381694 \\
\hline 6 & 0.918422 & 1.409254 & 2.346734 \\
\hline 6 & -0.090646 & 3.125890 & 3.726299 \\
\hline 1 & 1.954621 & 2.502248 & 3.901813 \\
\hline 6 & 2.063258 & 0.617951 & 1.862803 \\
\hline 7 & -0.264169 & 1.230481 & 1.682838 \\
\hline 6 & -1.291566 & 2.947533 & 3.027071 \\
\hline 1 & -0.017591 & 3.860966 & 4.519907 \\
\hline 6 & 3.336346 & 0.661490 & 2.447864 \\
\hline 7 & 1.830078 & -0.133155 & 0.746676 \\
\hline 6 & -1.336724 & 1.988549 & 2.019476 \\
\hline 1 & -2.171736 & 3.535531 & 3.256552 \\
\hline 6 & 4.380777 & -0.061179 & 1.868137 \\
\hline 1 & 3.517375 & 1.245011 & 3.340964 \\
\hline
\end{tabular}




$\begin{array}{llll}6 & 2.858628 & -0.799087 & 0.145411 \\ 1 & -2.241174 & 1.808980 & 1.451213 \\ 6 & 4.146871 & -0.784639 & 0.697167 \\ 1 & 5.368098 & -0.048558 & 2.314963 \\ 6 & 2.526835 & -1.459022 & -1.129416 \\ 1 & 4.957593 & -1.327794 & 0.229357 \\ 6 & 3.492393 & -2.034394 & -1.972193 \\ 7 & 1.211489 & -1.423832 & -1.503237 \\ 6 & 3.111928 & -2.564231 & -3.203670 \\ 1 & 4.534486 & -2.050846 & -1.680751 \\ 6 & 0.849631 & -1.933671 & -2.706624 \\ 6 & 1.764843 & -2.506896 & -3.584540 \\ 1 & 3.853587 & -3.004450 & -3.860705 \\ 1 & -0.203499 & -1.864992 & -2.950688 \\ 1 & 1.431214 & -2.896813 & -4.538453\end{array}$

Table S4. Cartesian coordinates for $\left(\mathrm{C}_{5} \mathrm{Me}_{5}\right)(\mathrm{tpy}) \mathrm{Lu}=\mathrm{CHSiMe}_{3}(8)$.

$\begin{array}{llll}71 & -0.004687 & -0.287480 & 0.185942 \\ 14 & -3.011729 & -1.643012 & -1.933446 \\ 6 & 1.420964 & -0.341435 & 2.486495 \\ 6 & 0.294060 & -499419 & 2.758804 \\ 6 & -1.242559 & -2.145966 & -1.289137 \\ 1 & -0.594050 & -1.668093 & -1.859476 \\ 6 & -0.484668 & -0.319828 & 2.451893 \\ 6 & -0.884229 & -1.680605 & 2.736470 \\ 6 & 0.940404 & -2.913814 & 2.301643 \\ 6 & 1.799147 & -2.726673 & 2.202583 \\ 1 & 2.734922 & -3.735638 & 1.664299 \\ 1 & 1.282223 & -3.285291 & 1.693036 \\ 1 & 2.074361 & -2.879874 & 3.201375 \\ 6 & -1.379421 & -3.686042 & 2.500508 \\ 1 & -1.010757 & -2.649230 & 1.857098 \\ 1 & -2.397620 & -3.285258 & 2.170432 \\ 1 & -1.450880 & 1.959324 & 3.521790 \\ 6 & 0.354665 & 2.471851 & 3.125435 \\ 1 & -0.590573 & & 2.915443\end{array}$




\begin{tabular}{|c|c|c|c|}
\hline 1 & 1.141295 & 2.489850 & 2.574609 \\
\hline 1 & 0.565566 & 2.100074 & 4.196180 \\
\hline 6 & 2.867463 & 0.076875 & 2.535382 \\
\hline 1 & 3.490227 & -0.511013 & 1.850506 \\
\hline 1 & 3.291050 & -0.053846 & 3.542785 \\
\hline 1 & 2.997599 & 1.132861 & 2.271064 \\
\hline 6 & -2.264930 & 0.100096 & 3.170405 \\
\hline 1 & -2.413502 & -0.083125 & 4.245341 \\
\hline 1 & -3.051906 & -0.456368 & 2.646467 \\
\hline 1 & -2.446257 & 1.167649 & 3.004481 \\
\hline 6 & -3.246344 & -3.211901 & -3.035261 \\
\hline 1 & -2.594286 & -3.167404 & -3.916024 \\
\hline 1 & -4.283796 & -3.295952 & -3.382485 \\
\hline 1 & -2.999339 & -4.121907 & -2.475359 \\
\hline 6 & -3.583825 & -0.137563 & -3.004441 \\
\hline 6 & -4.298579 & -1.811921 & -0.496420 \\
\hline 1 & -4.280525 & -0.946568 & 0.177251 \\
\hline 1 & -5.314202 & -1.901970 & -0.902450 \\
\hline 1 & -4.086824 & -2.706075 & 0.101228 \\
\hline 1 & -2.949192 & -0.041834 & -3.892654 \\
\hline 1 & -4.622198 & -0.274566 & -3.332610 \\
\hline 1 & -3.518292 & 0.799692 & -2.441303 \\
\hline 6 & -1.315669 & 4.100783 & -0.517792 \\
\hline 6 & -0.619618 & 2.877199 & -0.438244 \\
\hline 6 & -2.671244 & 4.152523 & -0.221910 \\
\hline 1 & -0.798942 & 5.000772 & -0.826685 \\
\hline 6 & 0.785712 & 2.718561 & -0.791129 \\
\hline 7 & -1.273128 & 1.725693 & -0.056347 \\
\hline 6 & -3.336192 & 2.967411 & 0.148336 \\
\hline 1 & -3.210935 & 5.090653 & -0.287394 \\
\hline 6 & 1.623724 & 3.786585 & -1.128080 \\
\hline 7 & 1.249345 & 1.414877 & -0.807109 \\
\hline 6 & -2.604371 & 1.791268 & 0.218787 \\
\hline 1 & -4.396292 & 2.961846 & 0.370565 \\
\hline 6 & 2.946606 & 3.541321 & -1.505551 \\
\hline 1 & 1.255404 & 4.804147 & -1.095437 \\
\hline 6 & 2.542119 & 1.171533 & -1.235529 \\
\hline
\end{tabular}




$\begin{array}{llll}1 & -3.074348 & 0.856467 & 0.492867 \\ 6 & 3.401641 & 2.219901 & -1.573183 \\ 1 & 3.607174 & 4.362750 & -1.756907 \\ 6 & 2.904739 & -0.238256 & -1.355008 \\ 1 & 2.018045 & -1.889268 \\ 6 & 4.417342 & -0.696243 & -1.929279 \\ 7 & 4.107188 & -1.134752 & -0.924858 \\ 6 & 1.953911 & -2.059178 & -2.093480 \\ 1 & 4.324790 & 0.011436 & -2.262781 \\ 6 & 4.855693 & -2.464233 & -1.107195 \\ 6 & 2.172048 & -2.966393 & -1.691237 \\ 1 & 3.326895 & -2.415784 & -2.537829 \\ 1 & 5.247434 & -3.120178 & -0.772048 \\ 1 & 1.378035 & -4.034344 & -1.824773\end{array}$

Table S5. Cartesian coordinates for $3 a$.

$\begin{array}{llll}71 & -0.547737 & 0.328278 & -0.086862 \\ 14 & -1.923404 & -1.858953 & 2.782735 \\ 7 & 1.026425 & 2.000490 & 0.605953 \\ 7 & 0.073838 & -1.182648 \\ 7 & 1.417538 & -1.600812 & -1.509185 \\ 6 & -0.664704 & 2.008909 & 0.050983 \\ 6 & 2.267175 & -0.710464 & -2.315706 \\ 6 & 1.434163 & -1.748866 & -2.375135 \\ 6 & 0.388098 & -3.764307 & -3.262980 \\ 6 & -0.622217 & 0.938491 & -0.979948 \\ 6 & 2.610089 & -0.439211 & -3.413595 \\ 6 & 2.236913 & 2.996067 & 0.420089 \\ 6 & 3.196023 & 0.836685 & -3.442442 \\ 6 & 2.898604 & -2.842805 & -3.259486 \\ 6 & 0.420938 & 3.971070 & 1.352227 \\ 6 & 2.844267 & 1.589986 & -2.316363 \\ 6 & 2.986337 & 3.947039 & 1.920499 \\ 6 & 1.563616 & 2.944576 & 1.524062 \\ 6 & 0.686957 & -3.590045 & -2.385702\end{array}$




\begin{tabular}{|c|c|c|c|}
\hline 6 & -1.684745 & -2.496522 & -1.528095 \\
\hline 6 & -1.038552 & -0.304128 & 2.141840 \\
\hline 1 & -0.056453 & -0.264701 & 2.653865 \\
\hline 1 & -1.610987 & 0.569943 & 2.517401 \\
\hline 14 & 3.817174 & -0.979678 & 1.123316 \\
\hline 6 & 3.848192 & 0.098804 & -0.487273 \\
\hline 1 & 4.714396 & 0.772584 & -0.410458 \\
\hline 1 & 4.086920 & -0.594439 & -1.305235 \\
\hline 6 & -2.034135 & -1.919045 & 4.709425 \\
\hline 1 & -1.032076 & -1.904916 & 5.155204 \\
\hline 1 & -2.545996 & -2.828744 & 5.048068 \\
\hline 1 & -2.586199 & -1.053017 & 5.094167 \\
\hline 6 & 3.295539 & -0.021473 & 2.704017 \\
\hline 1 & 2.238967 & 0.262645 & 2.686630 \\
\hline 1 & 3.894418 & 0.887279 & 2.832572 \\
\hline 1 & 3.456236 & -0.658176 & 3.582374 \\
\hline 6 & 2.734663 & -2.548727 & 0.917438 \\
\hline 1 & 2.885709 & -3.229139 & 1.764002 \\
\hline 1 & 3.003619 & -3.083571 & -0.000845 \\
\hline 1 & 1.671164 & -2.298594 & 0.863769 \\
\hline 71 & -0.547737 & 0.328278 & -0.086862 \\
\hline 14 & -1.923404 & -1.858953 & 2.782735 \\
\hline 7 & 1.026425 & 2.000490 & 0.605953 \\
\hline 7 & 1.417538 & 0.073838 & -1.182648 \\
\hline 7 & -0.664704 & -1.600812 & -1.509185 \\
\hline 6 & 2.267175 & 2.008909 & 0.050983 \\
\hline 6 & 1.434163 & -0.710464 & -2.315706 \\
\hline 6 & 0.388098 & -1.748866 & -2.375135 \\
\hline 6 & -0.622217 & -3.764307 & -3.262980 \\
\hline 6 & 2.610089 & 0.938491 & -0.979948 \\
\hline 6 & 2.236913 & -0.439211 & -3.413595 \\
\hline 6 & 3.196023 & 2.996067 & 0.420089 \\
\hline 6 & 2.898604 & 0.836685 & -3.442442 \\
\hline 6 & 0.420938 & -2.842805 & -3.259486 \\
\hline 6 & 2.844267 & 3.971070 & 1.352227 \\
\hline 6 & 2.986337 & 1.589986 & -2.316363 \\
\hline 6 & 1.563616 & 3.947039 & 1.920499 \\
\hline
\end{tabular}




$\begin{array}{llll}6 & 0.686957 & 2.944576 & 1.524062 \\ 6 & -1.703621 & -3.590045 & -2.385702 \\ 6 & -1.684745 & -2.496522 & -1.528095 \\ 6 & -1.038552 & -0.304128 & 2.141840 \\ 1 & -0.056453 & -0.264701 & 2.653865 \\ 1 & -1.610987 & 0.569943 & 2.517401 \\ 14 & 3.817174 & -0.979678 & 1.123316 \\ 6 & 3.848192 & 0.098804 & -0.487273 \\ 1 & 4.714396 & 0.772584 & -0.410458 \\ 1 & 4.086920 & -0.594439 & -1.305235 \\ 6 & -2.034135 & -1.919045 & 4.709425 \\ 1 & -1.032076 & -1.904916 & 5.155204 \\ 1 & -2.545996 & -2.828744 & 5.048068 \\ 1 & -2.586199 & -1.053017 & 5.094167 \\ 6 & 3.295539 & -0.021473 & 2.704017 \\ 1 & 2.238967 & 0.262645 & 2.686630 \\ 1 & 3.894418 & 0.887279 & 2.832572 \\ 1 & 3.456236 & -0.658176 & 3.582374 \\ 6 & 2.734663 & -2.548727 & 0.917438 \\ 1 & 2.885709 & -3.229139 & 1.764002 \\ 1 & 3.003619 & -3.083571 & -0.000845 \\ 1 & 1.671164 & -2.298594 & 0.863769\end{array}$

Table S6. Cartesian coordinates for (tpy) $\mathrm{Lu}\left(\mathrm{CH}_{2} \mathrm{SiMe}_{3}\right)_{3}(7)$.

$\begin{array}{llll}71 & 0.336897 & 0.049313 & -0.215663 \\ 14 & 3.048780 & -2.494207 & -1.202724 \\ 6 & 2.310252 & -0.750110 & -1.325951 \\ 1 & 2.032987 & -0.596270 & -2.389876 \\ 1 & 3.125717 & -0.036175 & -1.109698 \\ 14 & -2.788228 & -2.137668 & -1.464856 \\ 6 & -1.178457 & -1.178496 & -1.708901 \\ 1 & -0.400055 & -1.875752 & -2.067528 \\ 1 & -1.342036 & -0.439760 & -2.513601 \\ 6 & 4.328330 & -2.902599 & -2.590826 \\ 1 & 3.860785 & -2.842034 & -3.581216 \\ 1 & 4.737765 & -3.913541 & -2.468521\end{array}$




\begin{tabular}{|c|c|c|c|}
\hline 1 & 5.161826 & -2.190057 & -2.567319 \\
\hline 6 & -2.701788 & -3.437094 & -0.040994 \\
\hline 1 & -2.609394 & -2.961570 & 0.941276 \\
\hline 1 & -1.843543 & -4.106482 & -0.178043 \\
\hline 1 & -3.610756 & -4.051456 & -0.037784 \\
\hline 6 & -4.280436 & -0.970937 & -1.089537 \\
\hline 1 & -5.212515 & -1.548452 & -1.052357 \\
\hline 1 & -4.385592 & -0.211669 & -1.874359 \\
\hline 1 & -4.161772 & -0.454755 & -0.130539 \\
\hline 6 & 1.715041 & -3.887337 & -1.308882 \\
\hline 6 & 3.997417 & -2.733036 & 0.464203 \\
\hline 6 & -3.288237 & -3.137174 & -3.044268 \\
\hline 1 & -4.234572 & -3.672793 & -2.894664 \\
\hline 1 & -2.514229 & -3.873163 & -3.294199 \\
\hline 1 & -3.405047 & -2.467711 & -3.905146 \\
\hline 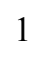 & 3.381629 & -2.499677 & 1.341638 \\
\hline 1 & 4.349040 & -3.767653 & 0.561185 \\
\hline 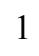 & 4.872687 & -2.073608 & 0.497466 \\
\hline 1 & 1.196659 & -3.854428 & -2.274759 \\
\hline 1 & 2.188636 & -4.872629 & -1.214037 \\
\hline 1 & 0.951906 & -3.797891 & -0.525945 \\
\hline 6 & -1.883618 & -1.475144 & 3.567873 \\
\hline 6 & -1.476768 & -0.780965 & 2.415916 \\
\hline 6 & -1.163937 & -2.587492 & 4.001468 \\
\hline 1 & -2.756668 & -1.156030 & 4.121731 \\
\hline 6 & -2.186329 & 0.404614 & 1.895887 \\
\hline 7 & -0.375993 & -1.196655 & 1.715543 \\
\hline 6 & -0.040123 & -3.002138 & 3.276244 \\
\hline 1 & -1.476066 & -3.124713 & 4.890031 \\
\hline 6 & -3.310975 & 0.959589 & 2.521427 \\
\hline 7 & -1.680082 & 0.952598 & 0.754431 \\
\hline 6 & 0.316433 & -2.281288 & 2.140473 \\
\hline 1 & 0.544235 & -3.861606 & 3.580396 \\
\hline 6 & -3.913251 & 2.089772 & 1.964812 \\
\hline 1 & -3.715309 & 0.528362 & 3.427713 \\
\hline 6 & -2.255666 & 2.061712 & 0.209029 \\
\hline 1 & 1.173041 & -2.565332 & 1.540627 \\
\hline
\end{tabular}




\begin{tabular}{|c|c|c|c|}
\hline 6 & -3.382810 & 2.648864 & 0.801483 \\
\hline 1 & -4.782280 & 2.533090 & 2.436909 \\
\hline 6 & -1.612702 & 2.594664 & -1.006813 \\
\hline 1 & -3.838582 & 3.533277 & 0.376456 \\
\hline 6 & -2.126677 & 3.682608 & -1.733090 \\
\hline 7 & -0.463507 & 1.972887 & -1.416516 \\
\hline 6 & -1.463348 & 4.135135 & -2.871977 \\
\hline 1 & -3.039989 & 4.170611 & -1.419182 \\
\hline 6 & 0.177688 & 2.420619 & -2.524232 \\
\hline 6 & -0.285634 & 3.494172 & -3.278153 \\
\hline 1 & -1.858620 & 4.972695 & -3.435728 \\
\hline 1 & 1.085341 & 1.893434 & -2.794095 \\
\hline 1 & 0.258885 & 3.816577 & -4.157077 \\
\hline 6 & 1.187330 & 1.727439 & 1.407712 \\
\hline 1 & 0.467726 & 2.561432 & 1.406329 \\
\hline 1 & 1.090497 & 1.224477 & 2.386684 \\
\hline 14 & 2.943426 & 2.437736 & 1.263350 \\
\hline 6 & 3.210807 & 3.924991 & 2.472526 \\
\hline 1 & 3.048811 & 3.614027 & 3.511639 \\
\hline 1 & 4.229968 & 4.324009 & 2.388867 \\
\hline 1 & 2.506922 & 4.736859 & 2.250089 \\
\hline 6 & 4.278217 & 1.124529 & 1.706669 \\
\hline 1 & 4.142845 & 0.766859 & 2.735009 \\
\hline 1 & 4.221020 & 0.260389 & 1.037094 \\
\hline 1 & 5.285624 & 1.551003 & 1.629333 \\
\hline 6 & 3.338842 & 3.126989 & -0.494683 \\
\hline 1 & 3.361417 & 2.325639 & -1.241960 \\
\hline 1 & 2.594273 & 3.869871 & -0.806605 \\
\hline 1 & 4.320869 & 3.616201 & -0.493677 \\
\hline
\end{tabular}

Table S7. Cartesian coordinates for (tpy) $\mathrm{Lu}\left(=\mathrm{CH}-\mathrm{SiMe}_{3}\right)\left(\mathrm{CH}_{2} \mathrm{SiMe}_{3}\right)(9)$.

$\begin{array}{llll}71 & -0.031273 & 0.048449 & -0.528404 \\ 14 & 3.377993 & -1.252964 & -1.825716 \\ 6 & 1.523334 & -1.259000 & -1.464617 \\ 1 & 1.093431 & -2.199518 & -1.871000 \\ 6 & 3.790677 & -1.967525 & -3.574396\end{array}$




\begin{tabular}{|c|c|c|c|}
\hline 1 & 3.427253 & -2.998249 & -3.665444 \\
\hline 1 & 4.874246 & -1.969695 & -3.748269 \\
\hline 1 & 3.317587 & -1.369786 & -4.362009 \\
\hline 6 & 4.361676 & -2.328769 & -0.551497 \\
\hline 6 & 4.144746 & 0.515086 & -1.748588 \\
\hline 1 & 4.069129 & 0.936674 & -0.739506 \\
\hline 1 & 5.206699 & 0.476327 & -2.021392 \\
\hline 1 & 3.640410 & 1.193935 & -2.446601 \\
\hline 1 & 4.014533 & -3.368200 & -0.574106 \\
\hline 1 & 5.434002 & -2.316628 & -0.785146 \\
\hline 1 & 4.222881 & -1.946021 & 0.465659 \\
\hline 6 & 2.056209 & 1.813975 & 3.196433 \\
\hline 6 & 1.269998 & 1.059104 & 2.303930 \\
\hline 6 & 2.800705 & 2.891830 & 2.731749 \\
\hline 1 & 2.083281 & 1.558044 & 4.247821 \\
\hline 6 & 0.434078 & -0.068592 & 2.702986 \\
\hline 7 & 1.248930 & 1.380548 & 0.963480 \\
\hline 6 & 2.758770 & 3.220476 & 1.363782 \\
\hline 1 & 3.405263 & 3.473621 & 3.418476 \\
\hline 6 & 0.300043 & -0.495762 & 4.027641 \\
\hline 7 & -0.249117 & -0.700406 & 1.676984 \\
\hline 6 & 1.974041 & 2.444673 & 0.523010 \\
\hline 1 & 3.323363 & 4.054954 & 0.966703 \\
\hline 6 & -0.547808 & -1.563355 & 4.334598 \\
\hline 1 & 0.843076 & 0.000267 & 4.822019 \\
\hline 6 & -1.113656 & -1.736217 & 1.988931 \\
\hline 1 & 1.910776 & 2.656749 & -0.538122 \\
\hline 6 & -1.266082 & -2.179804 & 3.304230 \\
\hline 1 & -0.657686 & -1.900671 & 5.358267 \\
\hline 6 & -1.838053 & -2.310839 & 0.858069 \\
\hline 1 & -1.941809 & -2.993529 & 3.535504 \\
\hline 6 & -2.701771 & -3.418594 & 0.949426 \\
\hline 7 & -1.624415 & -1.703536 & -0.358138 \\
\hline 6 & -3.342122 & -3.899010 & -0.187966 \\
\hline 1 & -2.865261 & -3.905242 & 1.902476 \\
\hline 6 & -2.259314 & -2.177935 & -1.463683 \\
\hline 6 & -3.117453 & -3.267832 & -1.425331 \\
\hline
\end{tabular}




$\begin{array}{llll}1 & -4.007255 & -4.752521 & -0.119214 \\ 1 & -2.057142 & -1.651480 & -2.389929 \\ 1 & -3.599210 & -3.615478 & -2.330889 \\ 1 & -4.080145 & 4.569634 & -1.902385 \\ 6 & -3.212827 & 4.065396 & -2.347698 \\ 14 & -2.607026 & 2.603992 & -1.240012 \\ 1 & -3.500826 & 3.701138 & -3.341283 \\ 1 & -2.417038 & 4.808474 & -2.480661 \\ 6 & -1.110351 & 1.661124 & -1.921843 \\ 6 & -4.115121 & 1.422218 & -1.010175 \\ 6 & -2.212689 & 3.362779 & 0.492947 \\ 1 & -1.402076 & 1.161360 & -2.865312 \\ 1 & -0.331344 & 2.395459 & -2.207845 \\ 1 & -3.878319 & 0.604322 & -0.318687 \\ 1 & -4.412088 & 0.978539 & -1.968599 \\ 1 & -4.977043 & 1.968626 & -0.608443 \\ 1 & -3.067030 & 3.940110 & 0.867128 \\ 1 & -1.348278 & 4.036203 & 0.439639 \\ 1 & -1.986477 & 2.581515 & 1.230084\end{array}$

Table S8. Cartesian coordinates for $\mathrm{SiMe}_{4}$.

$\begin{array}{llll}14 & 0.000899 & 0.000296 & -0.000868 \\ 6 & 1.155646 & 1.420694 & 0.578939 \\ 1 & 0.584781 & 2.206017 & 1.087804 \\ 1 & 1.914895 & 1.047492 & 1.276038 \\ 6 & -1.351890 & 0.708877 & -1.164857 \\ 1 & -0.902717 & 1.202096 & -2.034849 \\ 1 & -1.970702 & 1.445690 & -0.639618 \\ 1 & -2.010278 & -0.087952 & -1.529987 \\ 6 & 1.030566 & -1.319612 & -0.939633 \\ 1 & 0.393449 & -2.146496 & -1.274202 \\ 1 & 1.814536 & -1.735280 & -0.295792 \\ 1 & 1.513683 & -0.885126 & -1.822815 \\ 6 & -0.835313 & -0.810133 & 1.526656 \\ 1 & -1.443869 & -1.671402 & 1.227227 \\ 1 & -1.488978 & -0.095326 & 2.039931\end{array}$




$\begin{array}{llll}1 & -0.084616 & -1.159262 & 2.245174 \\ 1 & 1.673187 & 1.876456 & -0.273384\end{array}$

Figure S1. Molecular structure and numbering scheme of $3 \mathrm{~b}$ with thermal ellipsoids depicted at the $33 \%$ probability level.

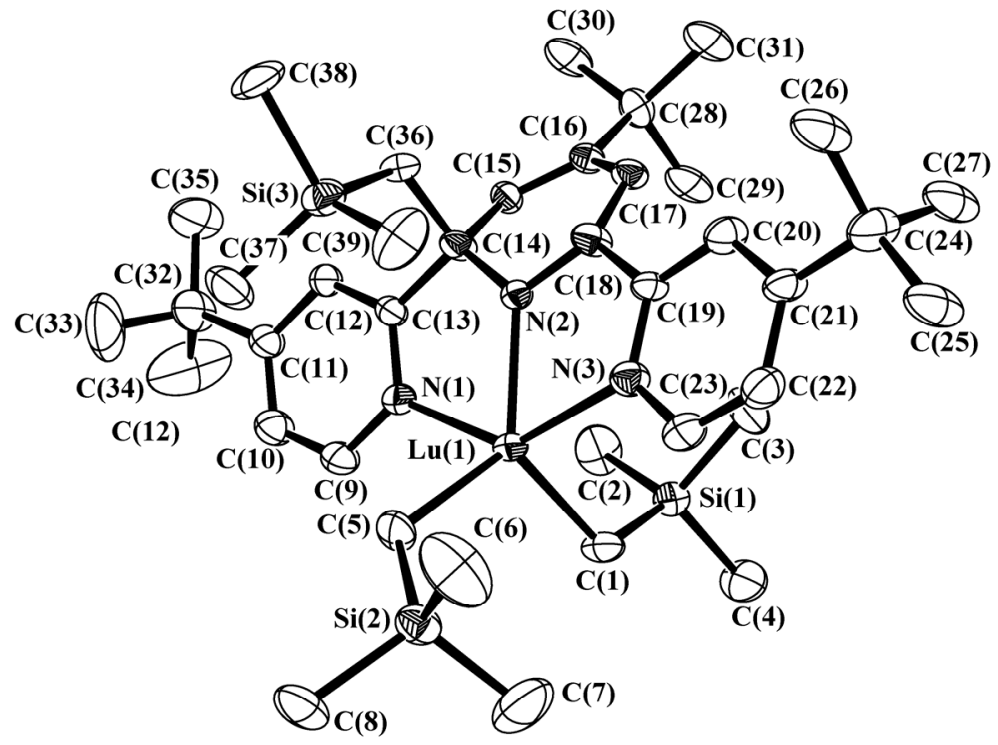

Crystallographic Details for 3b. An orange crystal grown overnight from a concentrated hexanes solution of $\mathbf{3 b}$ at $-30{ }^{\circ} \mathrm{C}$ was mounted from Paratone $\mathrm{N}$ oil (Hampton Research) onto a glass fiber under argon gas flow and placed on a Bruker P4/CCD diffractometer, equipped with a Bruker LT-2 temperature device. A hemisphere of data was collected using $\varphi$ scans, with $30 \mathrm{~s}$ frame exposures and $0.3^{\circ}$ frame widths. Data collection and initial indexing and cell refinement were handled using $\mathrm{SMART}^{4}$ software. Frame integration and final cell parameter calculations were carried out using $\mathrm{SAINT}^{5}$ software. The data was corrected for absorption using the SADABS program. ${ }^{6}$ Decay of reflection data was monitored by analysis of redundant frames. The structure was solved using Patterson techniques, completed by subsequent difference Fourier techniques, and refined by full-matrix least-squares procedures. Several disordered t-butyl methyl groups were refined in two one-half occupancy positions (C24, C25, C26, C28, C29, and 
C30). In addition, a disordered $\mathrm{CH}_{2} \mathrm{SiMe}_{3}$ group was refined in two positions, with siteoccupancy-factors allowed to vary for each position. The site-occupancy-factors of each position were tied to 1.0, and refined to an 80.7(7)\% contribution for position \#1 (Si3, C36, C37, C38, C39) and a 19.3(7)\% contribution for position \#2 (Si4, C40, C41, C42, C43). The temperature factors for position \#2 were refined isotropically. All non-hydrogen atoms were refined anisotropically, and hydrogen atoms were treated as idealized contributions. Hydrogen atom positions were not include on disordered carbon atoms. Structure solution, refinement, graphics, and creation of publication materials were performed using SHELXTL. ${ }^{7}$

Table S9. Crystal Data and Structure Refinement for $\mathbf{3 b .}$

\begin{tabular}{|c|c|c|}
\hline Identification code & \multicolumn{2}{|l|}{ data_tbuterpu2 } \\
\hline Empirical formula & \multicolumn{2}{|l|}{$\mathrm{C}_{39} \mathrm{H}_{68} \mathrm{~N}_{3} \mathrm{Lu} \mathrm{Si}_{3}$} \\
\hline Formula weight & \multicolumn{2}{|l|}{$838.21 \mathrm{~g} / \mathrm{mol}$} \\
\hline Temperature & \multicolumn{2}{|l|}{ 203(2) K } \\
\hline Wavelength & \multicolumn{2}{|l|}{$0.71073 \AA$} \\
\hline Crystal system & \multicolumn{2}{|l|}{ triclinic } \\
\hline Space group & \multicolumn{2}{|l|}{$P \overline{1}$} \\
\hline \multirow[t]{3}{*}{ Unit cell dimensions } & $\mathrm{a}=12.1805(16) \AA$ & $\alpha=90.928(2)^{\circ}$ \\
\hline & $\mathrm{b}=12.6262(18) \AA$ & $\beta=99.040(2)^{\circ}$ \\
\hline & $\mathrm{c}=15.275(2) \AA$ & $\gamma=97.883(3)^{\circ}$ \\
\hline Volume & \multicolumn{2}{|l|}{$2296.4(6) \AA^{3}$} \\
\hline $\mathrm{Z}$ & \multicolumn{2}{|l|}{2} \\
\hline Density (calculated) & \multicolumn{2}{|l|}{$1.287 \mathrm{~g} \mathrm{~cm}^{-3}$} \\
\hline Absorption coefficient & \multicolumn{2}{|l|}{$2.259 \mathrm{~mm}^{-1}$} \\
\hline $\mathrm{F}(000)$ & \multicolumn{2}{|l|}{916} \\
\hline Crystal size & \multicolumn{2}{|c|}{$0.24 \times 0.16 \times 0.10 \mathrm{~mm}^{3}$} \\
\hline Theta range for data collection & \multicolumn{2}{|l|}{1.35 to $28.17^{\circ}$} \\
\hline Index ranges & \multicolumn{2}{|c|}{$-15 \leq h \leq 12,-16 \leq k \leq 16,-19 \leq l \leq 19$} \\
\hline Reflections collected & \multicolumn{2}{|l|}{19530} \\
\hline Independent reflections & \multicolumn{2}{|l|}{$8764\left(R_{\text {int }}=0.0590\right)$} \\
\hline Max. and min. transmission & \multicolumn{2}{|l|}{0.8057 and 0.6132} \\
\hline Refinement method & \multicolumn{2}{|c|}{ full-matrix least-squares on $\mathrm{F}^{2}$} \\
\hline Data/restraints/parameters & \multicolumn{2}{|l|}{$8764 / 30 / 447$} \\
\hline Goodness-of-fit on $\mathrm{F}^{2}$ & \multicolumn{2}{|l|}{0.993} \\
\hline Final $R$ indices $[\mathrm{I}>2 \sigma(\mathrm{I})]$ & \multicolumn{2}{|c|}{$\mathrm{R} 1=0.0542, \mathrm{wR} 2=0.1251$} \\
\hline
\end{tabular}




\begin{tabular}{ll}
\hline $\mathrm{R}$ indices (all data) & $\mathrm{R} 1=0.1037, \mathrm{wR} 2=0.1423$ \\
Largest diff. peak and hole & 1.053 and $-0.877 \mathrm{e} . \AA^{-3}$ \\
\hline
\end{tabular}

Table S10. $\quad$ Bond lengths $(\AA)$ for $3 b$.

\begin{tabular}{|c|c|c|c|}
\hline Lu1-N2 & $2.217(7)$ & $\mathrm{C} 15-\mathrm{C} 16$ & $1.376(10)$ \\
\hline Lu1-C1 & $2.337(8)$ & $\mathrm{C} 16-\mathrm{C} 17$ & $1.415(11)$ \\
\hline Lu1-C5 & $2.346(9)$ & $\mathrm{C} 16-\mathrm{C} 28$ & $1.551(12)$ \\
\hline Lu1-N3 & $2.355(6)$ & C17-C18 & $1.387(12)$ \\
\hline Lu1-N1 & $2.360(7)$ & C18-C19 & $1.497(11)$ \\
\hline Si1-C1 & $1.843(9)$ & $\mathrm{C} 18-\mathrm{C} 40$ & $1.86(4)$ \\
\hline Si1-C3 & $1.869(10)$ & $\mathrm{C} 19-\mathrm{C} 20$ & $1.413(11)$ \\
\hline $\mathrm{Si} 1-\mathrm{C} 2$ & $1.871(10)$ & $\mathrm{C} 20-\mathrm{C} 21$ & $1.411(12)$ \\
\hline Sil-C4 & $1.905(10)$ & $\mathrm{C} 21-\mathrm{C} 22$ & $1.404(14)$ \\
\hline $\mathrm{Si} 2-\mathrm{C} 5$ & $1.842(10)$ & C21-C24 & $1.541(12)$ \\
\hline $\mathrm{Si} 2-\mathrm{C} 7$ & $1.879(13)$ & $\mathrm{C} 22-\mathrm{C} 23$ & $1.383(12)$ \\
\hline Si2-C6 & $1.897(12)$ & $\mathrm{C} 24-\mathrm{C} 25^{\prime}$ & $1.56(3)$ \\
\hline $\mathrm{Si} 2-\mathrm{C} 8$ & $1.903(10)$ & $\mathrm{C} 24-\mathrm{C} 27^{\prime}$ & $1.57(3)$ \\
\hline Si3-C41 & $1.29(11)$ & $\mathrm{C} 24-\mathrm{C} 25$ & $1.59(2)$ \\
\hline $\mathrm{Si3}-\mathrm{Si} 4$ & $1.742(16)$ & $\mathrm{C} 24-\mathrm{C} 26$ & $1.62(2)$ \\
\hline Si3-C36 & $1.879(11)$ & $\mathrm{C} 24-\mathrm{C} 27$ & $1.71(2)$ \\
\hline Si3-C37 & $1.895(13)$ & $\mathrm{C} 24-\mathrm{C} 26^{\prime}$ & $1.89(3)$ \\
\hline Si3-C39 & $1.899(16)$ & $\mathrm{C} 25-\mathrm{C} 25^{\prime}$ & $1.03(4)$ \\
\hline Si3-C38 & $1.897(12)$ & $\mathrm{C} 25-\mathrm{C} 26^{\prime}$ & $1.83(4)$ \\
\hline Si4-C39 & $1.10(2)$ & $\mathrm{C} 26-\mathrm{C} 27^{\prime}$ & $1.25(3)$ \\
\hline Si4-C42 & $1.85(4)$ & $\mathrm{C} 26-\mathrm{C} 26^{\prime}$ & $1.62(4)$ \\
\hline $\mathrm{Si} 4-\mathrm{C} 40$ & $1.87(4)$ & $\mathrm{C} 27^{\prime}-\mathrm{C} 27$ & $1.88(4)$ \\
\hline Si4-C43 & $1.90(4)$ & C28-C31 & $1.57(2)$ \\
\hline Si4-C41 & $1.95(5)$ & $\mathrm{C} 28-\mathrm{C} 31^{\prime}$ & $1.59(3)$ \\
\hline N1-C13 & $1.352(11)$ & $\mathrm{C} 28-\mathrm{C} 30$ & $1.59(2)$ \\
\hline N1-C9 & $1.375(10)$ & C28-C30' & $1.61(3)$ \\
\hline N2-C18 & $1.389(9)$ & $\mathrm{C} 28-\mathrm{C} 29$ & $1.67(2)$ \\
\hline N2-C14 & $1.444(10)$ & C28-C29' & $1.74(3)$ \\
\hline N3-C19 & $1.328(11)$ & C29-C29' & $1.02(3)$ \\
\hline $\mathrm{N} 3-\mathrm{C} 23$ & $1.379(11)$ & C29-C30' & $2.03(4)$ \\
\hline C9-C10 & $1.386(13)$ & C30-C30' & $1.08(4)$ \\
\hline C10-C11 & $1.364(14)$ & C31-C31' & $0.91(4)$ \\
\hline C11-C12 & $1.413(11)$ & $\mathrm{C} 27-\mathrm{C} 25^{\prime}$ & $1.97(4)$ \\
\hline
\end{tabular}




$\begin{array}{llll}\mathrm{C} 11-\mathrm{C} 32 & 1.539(13) & \mathrm{C} 32-\mathrm{C} 35 & 1.481(18) \\ \mathrm{C} 12-\mathrm{C} 13 & 1.405(11) & \mathrm{C} 32-\mathrm{C} 34 & 1.535(19) \\ \mathrm{C} 13-\mathrm{C} 14 & 1.519(11) & \mathrm{C} 32-\mathrm{C} 33 & 1.581(19) \\ \mathrm{C} 14-\mathrm{C} 15 & 1.466(12) & \mathrm{C} 38-\mathrm{C} 41 & 0.70(7) \\ \mathrm{C} 14-\mathrm{C} 36 & 1.665(12) & \mathrm{C} 39-\mathrm{C} 42 & 0.90(5)\end{array}$

Table S11. Bond Angles $\left(^{\circ}\right.$ ) for $3 b$.

\begin{tabular}{|c|c|c|c|}
\hline N2-Lu1-C1 & $122.5(3)$ & C21-C20-C19 & 118.4(9) \\
\hline N2-Lu1-C5 & 126.1(3) & $\mathrm{C} 22-\mathrm{C} 21-\mathrm{C} 20$ & $117.9(8)$ \\
\hline C1-Lu1-C5 & $111.0(4)$ & $\mathrm{C} 22-\mathrm{C} 21-\mathrm{C} 24$ & $121.9(8)$ \\
\hline N2-Lu1-N3 & $69.4(2)$ & C20-C21-C24 & 120.3(9) \\
\hline C1-Lu1-N3 & $98.4(3)$ & $\mathrm{C} 23-\mathrm{C} 22-\mathrm{C} 21$ & $120.8(8)$ \\
\hline C5-Lu1-N3 & $110.4(3)$ & $\mathrm{C} 22-\mathrm{C} 23-\mathrm{N} 3$ & $120.6(9)$ \\
\hline N2-Lu1-N1 & $69.4(2)$ & C21-C24-C25' & $106.4(12)$ \\
\hline C1-Lu1-N1 & $105.5(3)$ & C21-C24-C27' & $116.2(11)$ \\
\hline C5-Lu1-N1 & $91.9(3)$ & $\mathrm{C} 25^{\prime}-\mathrm{C} 24-\mathrm{C} 27^{\prime}$ & $128.7(17)$ \\
\hline N3-Lu1-N1 & $138.7(3)$ & $\mathrm{C} 21-\mathrm{C} 24-\mathrm{C} 25$ & $110.3(11)$ \\
\hline C1-Si1-C3 & $110.2(5)$ & $\mathrm{C} 25^{\prime}-\mathrm{C} 24-\mathrm{C} 25$ & $38.3(14)$ \\
\hline C1-Si1-C2 & $109.4(5)$ & $\mathrm{C} 27^{\prime}-\mathrm{C} 24-\mathrm{C} 25$ & $132.3(13)$ \\
\hline C3-Si1-C2 & 109.1(6) & $\mathrm{C} 21-\mathrm{C} 24-\mathrm{C} 26$ & $105.7(9)$ \\
\hline C1-Si1-C4 & $113.8(5)$ & $\mathrm{C} 25^{\prime}-\mathrm{C} 24-\mathrm{C} 26$ & $143.0(14)$ \\
\hline C3-Si1-C4 & $106.2(5)$ & $\mathrm{C} 27^{\prime}-\mathrm{C} 24-\mathrm{C} 26$ & $46.0(13)$ \\
\hline $\mathrm{C} 2-\mathrm{Si} 1-\mathrm{C} 4$ & $108.0(6)$ & $\mathrm{C} 25-\mathrm{C} 24-\mathrm{C} 26$ & $111.7(14)$ \\
\hline $\mathrm{C} 5-\mathrm{Si} 2-\mathrm{C} 7$ & 109.0(5) & $\mathrm{C} 21-\mathrm{C} 24-\mathrm{C} 27$ & $103.2(9)$ \\
\hline C5-Si2-C6 & $113.4(7)$ & $\mathrm{C} 25^{\prime}-\mathrm{C} 24-\mathrm{C} 27$ & $73.8(16)$ \\
\hline $\mathrm{C} 7-\mathrm{Si} 2-\mathrm{C} 6$ & 107.6(7) & $\mathrm{C} 27^{\prime}-\mathrm{C} 24-\mathrm{C} 27$ & $69.9(16)$ \\
\hline $\mathrm{C} 5-\mathrm{Si} 2-\mathrm{C} 8$ & $111.7(5)$ & $\mathrm{C} 25-\mathrm{C} 24-\mathrm{C} 27$ & $109.6(13)$ \\
\hline C7-Si2-C8 & $108.7(7)$ & $\mathrm{C} 26-\mathrm{C} 24-\mathrm{C} 27$ & $115.9(15)$ \\
\hline C6-Si2-C8 & $106.3(6)$ & C21-C24-C26' & 101.1(11) \\
\hline C41-Si3-Si4 & $79(3)$ & $\mathrm{C} 25^{\prime}-\mathrm{C} 24-\mathrm{C} 26^{\prime}$ & $101.3(16)$ \\
\hline C41-Si3-C36 & $101(6)$ & $\mathrm{C} 27^{\prime}-\mathrm{C} 24-\mathrm{C} 26^{\prime}$ & $97.3(15)$ \\
\hline Si4-Si3-C36 & $88.8(6)$ & C25-C24-C26' & $63.0(14)$ \\
\hline C41-Si3-C37 & $120(4)$ & C26-C24-C26' & $54.3(13)$ \\
\hline Si4-Si3-C37 & $146.9(9)$ & $\mathrm{C} 27-\mathrm{C} 24-\mathrm{C} 26^{\prime}$ & $155.6(11)$ \\
\hline C36-Si3-C37 & $111.8(6)$ & $\mathrm{C} 25^{\prime}-\mathrm{C} 25-\mathrm{C} 24$ & $69.6(19)$ \\
\hline C41-Si3-C39 & $100(4)$ & $\mathrm{C} 25^{\prime}-\mathrm{C} 25-\mathrm{C} 26^{\prime}$ & $136(3)$ \\
\hline Si4-Si3-C39 & $34.9(7)$ & $\mathrm{C} 24-\mathrm{C} 25-\mathrm{C} 26^{\prime}$ & $66.5(13)$ \\
\hline
\end{tabular}




\begin{tabular}{|c|c|c|c|}
\hline C36-Si3-C39 & $111.5(6)$ & $\mathrm{C} 27^{\prime}-\mathrm{C} 26-\mathrm{C} 26^{\prime}$ & $130(2)$ \\
\hline C37-Si3-C39 & $112.0(10)$ & $\mathrm{C} 27^{\prime}-\mathrm{C} 26-\mathrm{C} 24$ & $64.9(17)$ \\
\hline C41-Si3-C38 & $13(4)$ & $\mathrm{C} 266^{\prime} \mathrm{C} 26-\mathrm{C} 24$ & $71.3(14)$ \\
\hline Si4-Si3-C38 & $90.8(7)$ & $\mathrm{C} 26-\mathrm{C} 27^{\prime}-\mathrm{C} 24$ & $69.2(18)$ \\
\hline C36-Si3-C38 & $104.9(7)$ & $\mathrm{C} 26-\mathrm{C} 27^{\prime}-\mathrm{C} 27$ & $128(2)$ \\
\hline C37-Si3-C38 & $107.4(7)$ & $\mathrm{C} 24-\mathrm{C} 27^{\prime}-\mathrm{C} 27$ & $58.5(12)$ \\
\hline C39-Si3-C38 & $108.9(8)$ & $\mathrm{C} 16-\mathrm{C} 28-\mathrm{C} 31$ & $112.9(11)$ \\
\hline C39-Si4-Si3 & $80.3(13)$ & $\mathrm{C} 16-\mathrm{C} 28-\mathrm{C} 31^{\prime}$ & $105.7(12)$ \\
\hline C39-Si4-C42 & $20(2)$ & C31-C28-C31' & $33.4(14)$ \\
\hline Si3-Si4-C42 & $98(2)$ & $\mathrm{C} 16-\mathrm{C} 28-\mathrm{C} 30$ & 109.2(9) \\
\hline C39-Si4-C40 & $126.0(18)$ & $\mathrm{C} 31-\mathrm{C} 28-\mathrm{C} 30$ & $109.6(14)$ \\
\hline $\mathrm{Si3}-\mathrm{Si} 4-\mathrm{C} 40$ & 104.1(13) & $\mathrm{C} 31^{\prime}-\mathrm{C} 28-\mathrm{C} 30$ & $82.6(17)$ \\
\hline C42-Si4-C40 & $113(2)$ & $\mathrm{C} 16-\mathrm{C} 28-\mathrm{C} 30^{\prime}$ & $109.8(11)$ \\
\hline C39-Si4-C43 & $113(3)$ & $\mathrm{C} 31-\mathrm{C} 28-\mathrm{C} 30^{\prime}$ & $134.6(15)$ \\
\hline Si3-Si4-C43 & $125(2)$ & $\mathrm{C} 31^{\prime}-\mathrm{C} 28-\mathrm{C} 30^{\prime}$ & 119.0(19) \\
\hline C42-Si4-C43 & $108(3)$ & $\mathrm{C} 30-\mathrm{C} 28-\mathrm{C} 30^{\prime}$ & $39.6(15)$ \\
\hline C40-Si4-C43 & $108(3)$ & $\mathrm{C} 16-\mathrm{C} 28-\mathrm{C} 29$ & $103.9(10)$ \\
\hline C39-Si4-C41 & $105(4)$ & C31-C28-C29 & $106.9(12)$ \\
\hline Si3-Si4-C41 & $40(4)$ & $\mathrm{C} 31^{\prime}-\mathrm{C} 28-\mathrm{C} 29$ & $138.2(15)$ \\
\hline C42-Si4-C41 & $124(4)$ & $\mathrm{C} 30-\mathrm{C} 28-\mathrm{C} 29$ & $114.4(12)$ \\
\hline C40-Si4-C41 & $113(4)$ & $\mathrm{C} 30^{\prime}-\mathrm{C} 28-\mathrm{C} 29$ & $76.4(16)$ \\
\hline C43-Si4-C41 & $85(4)$ & $\mathrm{C} 16-\mathrm{C} 28-\mathrm{C} 29^{\prime}$ & $101.9(11)$ \\
\hline C13-N1-C9 & $119.2(7)$ & C31-C28-C29' & $76.2(14)$ \\
\hline C13-N1-Lu1 & $119.0(5)$ & C31'-C28-C29' & $109.7(16)$ \\
\hline C9-N1-Lu1 & $121.6(6)$ & $\mathrm{C} 30-\mathrm{C} 28-\mathrm{C} 29^{\prime}$ & $142.1(13)$ \\
\hline C18-N2-C14 & $111.2(7)$ & $\mathrm{C} 30^{\prime}-\mathrm{C} 28-\mathrm{C} 29^{\prime}$ & $109.4(17)$ \\
\hline C18-N2-Lu1 & $122.3(5)$ & C29-C28-C29' & $34.6(11)$ \\
\hline C14-N2-Lu1 & $125.5(5)$ & C29'-C29-C28 & $76(2)$ \\
\hline C19-N3-C23 & $119.6(7)$ & C29'-C29-C30' & $124(3)$ \\
\hline C19-N3-Lu1 & $118.0(5)$ & C28-C29-C30' & $50.5(10)$ \\
\hline C23-N3-Lu1 & $122.4(7)$ & $\mathrm{C} 30^{\prime}-\mathrm{C} 30-\mathrm{C} 28$ & $71(2)$ \\
\hline Si1-C1-Lu1 & $118.3(5)$ & $\mathrm{C} 31^{\prime}-\mathrm{C} 31-\mathrm{C} 28$ & $74(2)$ \\
\hline $\mathrm{Si} 2-\mathrm{C} 5-\mathrm{Lu} 1$ & $126.7(4)$ & $\mathrm{C} 24-\mathrm{C} 27-\mathrm{C} 27^{\prime}$ & $51.6(11)$ \\
\hline N1-C9-C10 & $120.4(9)$ & $\mathrm{C} 24-\mathrm{C} 27-\mathrm{C} 25^{\prime}$ & $49.7(10)$ \\
\hline C11-C10-C9 & 121.1(8) & $\mathrm{C} 27^{\prime}-\mathrm{C} 27-\mathrm{C} 25^{\prime}$ & $94.4(15)$ \\
\hline $\mathrm{C} 10-\mathrm{C} 11-\mathrm{C} 12$ & 119.1(8) & $\mathrm{C} 26-\mathrm{C} 26^{\prime}-\mathrm{C} 25$ & $100.4(17)$ \\
\hline C10-C11-C32 & $120.8(8)$ & $\mathrm{C} 26-\mathrm{C} 26^{\prime}-\mathrm{C} 24$ & $54.4(12)$ \\
\hline
\end{tabular}




\begin{tabular}{|c|c|c|c|}
\hline C12-C11-C32 & $120.0(10)$ & $\mathrm{C} 25-\mathrm{C} 26^{\prime}-\mathrm{C} 24$ & $50.5(10)$ \\
\hline C13-C12-C11 & $118.0(9)$ & $\mathrm{C} 30-\mathrm{C} 30^{\prime}-\mathrm{C} 28$ & $69.4(18)$ \\
\hline N1-C13-C12 & $122.1(7)$ & $\mathrm{C} 30-\mathrm{C} 30^{\prime}-\mathrm{C} 29$ & $120(2)$ \\
\hline N1-C13-C14 & $117.3(7)$ & $\mathrm{C} 28-\mathrm{C} 30^{\prime}-\mathrm{C} 29$ & $53.1(11)$ \\
\hline C12-C13-C14 & $120.6(8)$ & $\mathrm{C} 25-\mathrm{C} 25^{\prime}-\mathrm{C} 24$ & $72(2)$ \\
\hline N2-C14-C15 & $114.5(6)$ & $\mathrm{C} 25-\mathrm{C} 25^{\prime}-\mathrm{C} 27$ & $125(3)$ \\
\hline N2-C14-C13 & $108.7(8)$ & $\mathrm{C} 24-\mathrm{C} 25^{\prime}-\mathrm{C} 27$ & $56.5(13)$ \\
\hline C15-C14-C13 & 112.1(7) & $\mathrm{C} 29-\mathrm{C} 29^{\prime}-\mathrm{C} 28$ & $69(2)$ \\
\hline N2-C14-C36 & $106.7(7)$ & C31-C31'-C28 & $72(2)$ \\
\hline C15-C14-C36 & $106.5(8)$ & C35-C32-C11 & $113.4(8)$ \\
\hline C13-C14-C36 & $108.1(6)$ & C35-C32-C34 & $116.5(14)$ \\
\hline C16-C15-C14 & $118.8(7)$ & C11-C32-C34 & $110.2(11)$ \\
\hline C15-C16-C17 & $118.5(8)$ & C35-C32-C33 & $105.5(12)$ \\
\hline C15-C16-C28 & 121.2(7) & C11-C32-C33 & $105.3(11)$ \\
\hline C17-C16-C28 & $119.9(7)$ & C34-C32-C33 & $104.7(14)$ \\
\hline C18-C17-C16 & $118.7(7)$ & C14-C36-Si3 & $122.2(7)$ \\
\hline N2-C18-C17 & $123.3(7)$ & C41-C38-Si3 & $24(10)$ \\
\hline N2-C18-C19 & 111.3(8) & C42-C39-Si4 & $135(5)$ \\
\hline C17-C18-C19 & 121.2(7) & C42-C39-Si3 & $152(5)$ \\
\hline N2-C18-C40 & $96.6(12)$ & Si4-C39-Si3 & $64.7(12)$ \\
\hline C17-C18-C40 & $90.0(14)$ & C18-C40-Si4 & $125(2)$ \\
\hline C19-C18-C40 & $104.6(13)$ & C38-C41-Si3 & $143(10)$ \\
\hline N3-C19-C20 & $122.7(7)$ & C38-C41-Si4 & $153(10)$ \\
\hline N3-C19-C18 & $116.3(7)$ & $\mathrm{Si3}-\mathrm{C} 41-\mathrm{Si} 4$ & $61(2)$ \\
\hline C20-C19-C18 & $120.9(8)$ & C39-C42-Si4 & $25(3)$ \\
\hline
\end{tabular}


Figure S2. Molecular structure and numbering scheme of $5 \mathrm{~b}$ with thermal ellipsoids depicted at the $33 \%$ probability level.

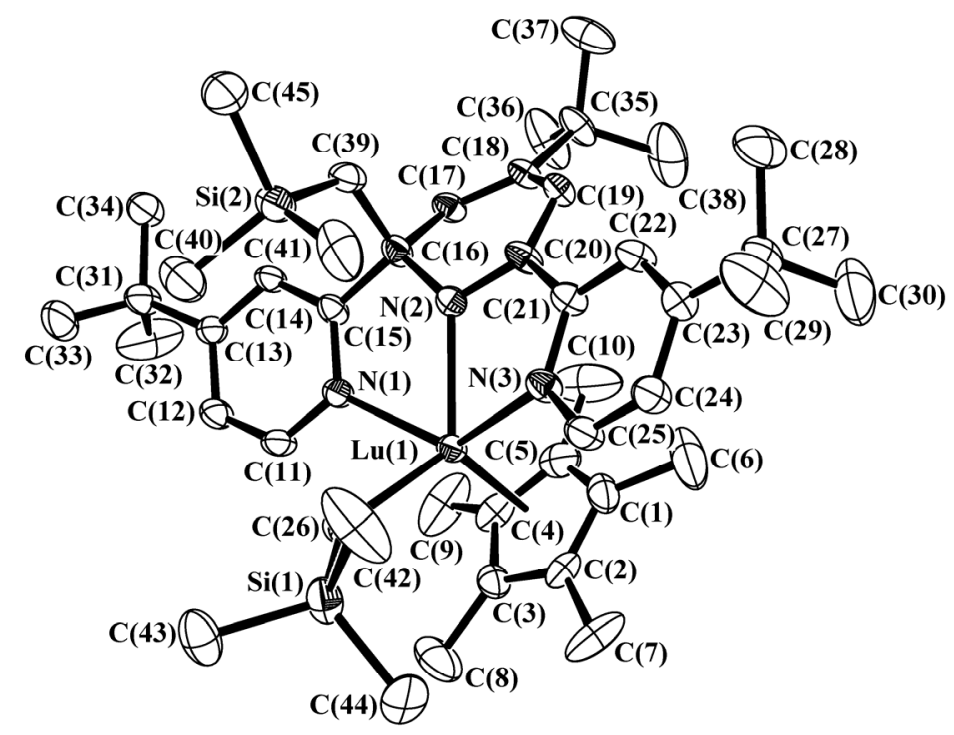

Crystallographic Details for 5b. An orange crystal grown overnight from a concentrated pentane solution of $\mathbf{5 b}$ at $-30{ }^{\circ} \mathrm{C}$ was mounted from Paratone $\mathrm{N}$ oil (Hampton Research) onto a glass fiber under argon gas flow and placed on a Bruker P4/CCD diffractometer, equipped with a Bruker LT-2 temperature device. A hemisphere of data was collected using $\varphi$ scans, with $30 \mathrm{~s}$ frame exposures and $0.3^{\circ}$ frame widths. Data collection and initial indexing and cell refinement were handled using SMART software. Frame integration and final cell parameter calculations were carried out using SAINT software. The data was corrected for absorption using the SADABS program. Decay of reflection data was monitored by analysis of redundant frames. The structure was solved using Patterson techniques, completed by subsequent difference Fourier techniques, and refined by full-matrix least-squares procedures. Carbon atom C44 had an unusually large and elongated atomic-displacement parameter, and was modeled as two one-half occupancy sites. All non-hydrogen atoms were refined anisotropically, and hydrogen atoms were treated as idealized contributions. Hydrogen atom positions on disordered atom C44 were not included in the refinement. Structure solution, refinement, graphics, and creation of publication materials were performed using SHELXTL. ${ }^{7}$ 
Table S12. Crystal Data and Structure Refinement for $\mathbf{5 b}$.

\begin{tabular}{|c|c|c|}
\hline Identification code & \multicolumn{2}{|l|}{ data_lucptmsa } \\
\hline Empirical formula & \multicolumn{2}{|l|}{$\mathrm{C}_{45} \mathrm{H}_{72} \mathrm{~N}_{3} \mathrm{Lu} \mathrm{Si}_{2}$} \\
\hline Formula weight & \multicolumn{2}{|l|}{$886.23 \mathrm{~g} / \mathrm{mol}$} \\
\hline Temperature & \multicolumn{2}{|l|}{$203(2) \mathrm{K}$} \\
\hline Wavelength & \multicolumn{2}{|l|}{$0.71073 \AA$} \\
\hline Crystal system & \multicolumn{2}{|l|}{ triclinic } \\
\hline Space group & \multicolumn{2}{|l|}{$P \overline{1}$} \\
\hline \multirow[t]{3}{*}{ Unit cell dimensions } & $\mathrm{a}=10.7538(14) \AA$ & $\alpha=98.937(2)^{\circ}$ \\
\hline & $\mathrm{b}=11.8122(16) \AA$ & $\beta=92.135(3)^{\circ}$ \\
\hline & $\mathrm{c}=20.525(3) \AA$ & $\gamma=113.937(2)^{\circ}$ \\
\hline Volume & \multicolumn{2}{|l|}{$2339.4(5) \AA^{3}$} \\
\hline $\mathrm{Z}$ & \multicolumn{2}{|l|}{2} \\
\hline Density (calculated) & \multicolumn{2}{|l|}{$1.224 \mathrm{~g} \mathrm{~cm}^{-3}$} \\
\hline Absorption coefficient & \multicolumn{2}{|l|}{$2.190 \mathrm{~mm}^{-1}$} \\
\hline $\mathrm{F}(000)$ & \multicolumn{2}{|l|}{888} \\
\hline Crystal size & \multicolumn{2}{|c|}{$0.20 \times 0.12 \times 0.10 \mathrm{~mm}^{3}$} \\
\hline Theta range for data collection & \multicolumn{2}{|l|}{$1.92-28.37^{\circ}$} \\
\hline Index ranges & \multicolumn{2}{|c|}{$-14 \leq h \leq 13,-15 \leq k \leq 15,-27 \leq l \leq 26$} \\
\hline Reflections collected & \multicolumn{2}{|l|}{23625} \\
\hline Independent reflections & \multicolumn{2}{|l|}{$9521\left(R_{\mathrm{int}}=0.0510\right)$} \\
\hline Max. and min. transmission & \multicolumn{2}{|l|}{0.8107 and 0.6684} \\
\hline Refinement method & \multicolumn{2}{|c|}{ full-matrix least-squares on $\mathrm{F}^{2}$} \\
\hline Data/restraints/parameters & \multicolumn{2}{|l|}{$9521 / 0 / 519$} \\
\hline Goodness-of-fit on $\mathrm{F}^{2}$ & \multicolumn{2}{|l|}{0.974} \\
\hline Final $R$ indices $[\mathrm{I}>2 \sigma(\mathrm{I})]$ & \multicolumn{2}{|c|}{$\mathrm{R} 1=0.0438, \mathrm{wR} 2=0.0779$} \\
\hline $\mathrm{R}$ indices (all data) & \multicolumn{2}{|c|}{$\mathrm{R} 1=0.0770, \mathrm{wR} 2=0.0857$} \\
\hline Largest diff. peak and hole & \multicolumn{2}{|c|}{1.130 and -0.658 e. $\AA^{-3}$} \\
\hline
\end{tabular}

Table S13. Bond lengths ( $\AA$ ) for $5 \mathrm{~b}$.

$\begin{array}{llll}\mathrm{Lu}(1)-\mathrm{N}(2) & 2.253(4) & \mathrm{C}(18)-\mathrm{C}(17) & 1.358(6) \\ \mathrm{Lu}(1)-\mathrm{C}(26) & 2.379(5) & \mathrm{C}(18)-\mathrm{C}(35) & 1.544(6) \\ \mathrm{Lu}(1)-\mathrm{N}(3) & 2.381(4) & \mathrm{C}(13)-\mathrm{C}(12) & 1.402(7) \\ \mathrm{Lu}(1)-\mathrm{N}(1) & 2.387(4) & \mathrm{C}(13)-\mathrm{C}(31) & 1.530(7) \\ \mathrm{Lu}(1)-\mathrm{C}(2) & 2.601(6) & \mathrm{C}(12)-\mathrm{C}(11) & 1.359(7)\end{array}$




$\begin{array}{llll}\mathrm{Lu}(1)-\mathrm{C}(1) & 2.606(6) & \mathrm{C}(24)-\mathrm{C}(25) & 1.371(7) \\ \mathrm{Lu}(1)-\mathrm{C}(5) & 2.613(6) & \mathrm{C}(5)-\mathrm{C}(1) & 1.369(9) \\ \mathrm{Lu}(1)-\mathrm{C}(3) & 2.622(5) & \mathrm{C}(5)-\mathrm{C}(4) & 1.378(8) \\ \mathrm{Lu}(1)-\mathrm{C}(4) & 2.624(5) & \mathrm{C}(5)-\mathrm{C}(10) & 1.516(8) \\ \mathrm{Si}(1)-\mathrm{C}(44) & 1.81(3) & \mathrm{C}(1)-\mathrm{C}(2) & 1.390(8) \\ \mathrm{Si}(1)-\mathrm{C}(26) & 1.816(5) & \mathrm{C}(1)-\mathrm{C}(6) & 1.519(8) \\ \mathrm{Si}(1)-\mathrm{C}\left(42^{\prime}\right) & 1.84(4) & \mathrm{C}(3)-\mathrm{C}(4) & 1.411(8) \\ \mathrm{Si}(1)-\mathrm{C}(43) & 1.852(6) & \mathrm{C}(3)-\mathrm{C}(2) & 1.414(7) \\ \mathrm{Si}(1)-\mathrm{C}(42 & 1.95(4) & \mathrm{C}(3)-\mathrm{C}(8) & 1.511(7) \\ \mathrm{Si}(1)-\mathrm{C}\left(44^{\prime}\right) & 2.00(3) & \mathrm{C}(2)-\mathrm{C}(7) & 1.515(8) \\ \mathrm{N}(1)-\mathrm{C}(15) & 1.349(6) & \mathrm{C}(4)-\mathrm{C}(9) & 1.523(8) \\ \mathrm{N}(1)-\mathrm{C}(11) & 1.350(6) & \mathrm{C}(27)-\mathrm{C}(30) & 1.473(9) \\ \mathrm{N}(2)-\mathrm{C}(20) & 1.365(5) & \mathrm{C}(27)-\mathrm{C}(28) & 1.511(8) \\ \mathrm{N}(2)-\mathrm{C}(16) & 1.447(6) & \mathrm{C}(27)-\mathrm{C}(29) & 1.523(8) \\ \mathrm{N}(3)-\mathrm{C}(25) & 1.347(6) & \mathrm{C}(35)-\mathrm{C}(37) & 1.50(3) \\ \mathrm{N}(3)-\mathrm{C}(21) & 1.350(6) & \mathrm{C}(35)-\mathrm{C}(36) & 1.503(7) \\ \mathrm{C}(15)-\mathrm{C}(14) & 1.388(7) & \mathrm{C}(35)-\mathrm{C}(38) & 1.526(9) \\ \mathrm{C}(15)-\mathrm{C}(16) & 1.517(6) & \mathrm{C}(35)-\mathrm{C}\left(37^{\prime}\right) & 1.59(4) \\ \mathrm{C}(20)-\mathrm{C}(19) & 1.376(6) & \mathrm{C}(34)-\mathrm{C}(31) & 1.512(7) \\ \mathrm{C}(20)-\mathrm{C}(21) & 1.462(6) & \mathrm{C}(31)-\mathrm{C}(32) & 1.513(9) \\ \mathrm{C}(21)-\mathrm{C}(22) & 1.394(6) & \mathrm{C}(31)-\mathrm{C}(33) & 1.545(9) \\ \mathrm{C}(23)-\mathrm{C}(22) & 1.383(7) & \left.\mathrm{C}(37)^{\prime}\right)-\mathrm{C}(37) & 0.40(8) \\ \mathrm{C}(23)-\mathrm{C}(24) & 1.392(7) & \mathrm{Si}(2)-\mathrm{C}(41) & 1.791(9) \\ \mathrm{C}(23)-\mathrm{C}(27) & 1.531(6) & \mathrm{Si}(2)-\mathrm{C}(40) & 1.847(6) \\ \mathrm{C}(16)-\mathrm{C}(17) & 1.496(6) & \mathrm{Si}(2)-\mathrm{C}(45) & 1.862(7) \\ \mathrm{C}(16)-\mathrm{C}(39) & 1.604(8) & \mathrm{Si}(2)-\mathrm{C}(39) & 1.879(5) \\ \mathrm{C}(19)-\mathrm{C}(18) & 1.439(6) & \mathrm{C}(42)-\mathrm{C}\left(42^{\prime}\right) & 0.64(8) \\ \mathrm{C}(14)-\mathrm{C}(13) & 1.377(6) & & \end{array}$

Table S14. Angles $\left(^{\circ}\right.$ ) for $5 b$.

$\begin{array}{llll}\mathrm{N}(2)-\mathrm{Lu}(1)-\mathrm{C}(26) & 119.66(17) & \mathrm{C}(13)-\mathrm{C}(14)-\mathrm{C}(15) & 122.1(5) \\ \mathrm{N}(2)-\mathrm{Lu}(1)-\mathrm{N}(3) & 68.04(14) & \mathrm{C}(17)-\mathrm{C}(18)-\mathrm{C}(19) & 117.6(4) \\ \mathrm{C}(26)-\mathrm{Lu}(1)-\mathrm{N}(3) & 101.19(15) & \mathrm{C}(17)-\mathrm{C}(18)-\mathrm{C}(35) & 123.0(4) \\ \mathrm{N}(2)-\mathrm{Lu}(1)-\mathrm{N}(1) & 68.01(13) & \mathrm{C}(19)-\mathrm{C}(18)-\mathrm{C}(35) & 119.3(4) \\ \mathrm{C}(26)-\mathrm{Lu}(1)-\mathrm{N}(1) & 87.54(16) & \mathrm{C}(23)-\mathrm{C}(22)-\mathrm{C}(21) & 121.6(5)\end{array}$




\begin{tabular}{|c|c|c|c|}
\hline $\mathrm{N}(3)-\mathrm{Lu}(1)-\mathrm{N}(1)$ & $133.43(15)$ & $\mathrm{C}(14)-\mathrm{C}(13)-\mathrm{C}(12)$ & $115.9(5)$ \\
\hline $\mathrm{N}(2)-\mathrm{Lu}(1)-\mathrm{C}(2)$ & $136.60(16)$ & $C(14)-C(13)-C(31)$ & $124.3(4)$ \\
\hline $\mathrm{C}(26)-\mathrm{Lu}(1)-\mathrm{C}(2)$ & $101.46(19)$ & $\mathrm{C}(12)-\mathrm{C}(13)-\mathrm{C}(31)$ & $119.7(4)$ \\
\hline $\mathrm{N}(3)-\mathrm{Lu}(1)-\mathrm{C}(2)$ & $92.21(16)$ & $C(18)-C(17)-C(16)$ & $118.9(4)$ \\
\hline $\mathrm{N}(1)-\mathrm{Lu}(1)-\mathrm{C}(2)$ & $131.13(15)$ & $\mathrm{C}(11)-\mathrm{C}(12)-\mathrm{C}(13)$ & $119.9(5)$ \\
\hline $\mathrm{N}(2)-\mathrm{Lu}(1)-\mathrm{C}(1)$ & $105.75(17)$ & $\mathrm{N}(1)-\mathrm{C}(11)-\mathrm{C}(12)$ & $123.8(5)$ \\
\hline $\mathrm{C}(26)-\mathrm{Lu}(1)-\mathrm{C}(1)$ & $131.6(2)$ & $C(25)-C(24)-C(23)$ & $120.0(5)$ \\
\hline $\mathrm{N}(3)-\mathrm{Lu}(1)-\mathrm{C}(1)$ & $79.82(17)$ & $\mathrm{C}(1)-\mathrm{C}(5)-\mathrm{C}(4)$ & $109.0(5)$ \\
\hline $\mathrm{N}(1)-\mathrm{Lu}(1)-\mathrm{C}(1)$ & $126.88(18)$ & $C(1)-C(5)-C(10)$ & $123.8(7)$ \\
\hline $\mathrm{C}(2)-\mathrm{Lu}(1)-\mathrm{C}(1)$ & $30.97(18)$ & $C(4)-C(5)-C(10)$ & $127.1(7)$ \\
\hline $\mathrm{N}(2)-\mathrm{Lu}(1)-\mathrm{C}(5)$ & $93.82(17)$ & $C(1)-C(5)-L u(1)$ & $74.5(4)$ \\
\hline$C(26)-\mathrm{Lu}(1)-\mathrm{C}(5)$ & $144.9(2)$ & $C(4)-C(5)-\operatorname{Lu}(1)$ & $75.2(3)$ \\
\hline $\mathrm{N}(3)-\mathrm{Lu}(1)-\mathrm{C}(5)$ & $100.97(19)$ & $C(10)-C(5)-L u(1)$ & $119.7(5)$ \\
\hline $\mathrm{N}(1)-\mathrm{Lu}(1)-\mathrm{C}(5)$ & $96.53(19)$ & $\mathrm{N}(3)-\mathrm{C}(25)-\mathrm{C}(24)$ & $123.6(5)$ \\
\hline $\mathrm{C}(2)-\mathrm{Lu}(1)-\mathrm{C}(5)$ & $50.84(18)$ & $C(5)-C(1)-C(2)$ & $108.4(5)$ \\
\hline $\mathrm{C}(1)-\mathrm{Lu}(1)-\mathrm{C}(5)$ & $30.41(19)$ & $C(5)-C(1)-C(6)$ & $124.2(7)$ \\
\hline $\mathrm{N}(2)-\mathrm{Lu}(1)-\mathrm{C}(3)$ & $143.08(15)$ & $C(2)-C(1)-C(6)$ & $126.9(7)$ \\
\hline $\mathrm{C}(26)-\mathrm{Lu}(1)-\mathrm{C}(3)$ & $93.77(18)$ & $\mathrm{C}(5)-\mathrm{C}(1)-\mathrm{Lu}(1)$ & $75.1(4)$ \\
\hline $\mathrm{N}(3)-\mathrm{Lu}(1)-\mathrm{C}(3)$ & $123.64(16)$ & $C(2)-C(1)-L u(1)$ & $74.3(3)$ \\
\hline $\mathrm{N}(1)-\mathrm{Lu}(1)-\mathrm{C}(3)$ & $100.89(16)$ & $\mathrm{C}(6)-\mathrm{C}(1)-\mathrm{Lu}(1)$ & $122.7(4)$ \\
\hline $\mathrm{C}(2)-\mathrm{Lu}(1)-\mathrm{C}(3)$ & $31.42(16)$ & $\mathrm{Si}(1)-\mathrm{C}(26)-\mathrm{Lu}(1)$ & $141.5(3)$ \\
\hline $\mathrm{C}(1)-\mathrm{Lu}(1)-\mathrm{C}(3)$ & $51.47(17)$ & $\mathrm{C}(4)-\mathrm{C}(3)-\mathrm{C}(2)$ & $106.1(5)$ \\
\hline $\mathrm{C}(5)-\mathrm{Lu}(1)-\mathrm{C}(3)$ & $51.20(17)$ & $\mathrm{C}(4)-\mathrm{C}(3)-\mathrm{C}(8)$ & $127.1(6)$ \\
\hline $\mathrm{N}(2)-\mathrm{Lu}(1)-\mathrm{C}(4)$ & $112.47(16)$ & $\mathrm{C}(2)-\mathrm{C}(3)-\mathrm{C}(8)$ & $126.5(6)$ \\
\hline $\mathrm{C}(26)-\mathrm{Lu}(1)-\mathrm{C}(4)$ & $117.5(2)$ & $C(4)-C(3)-L u(1)$ & $74.5(3)$ \\
\hline $\mathrm{N}(3)-\mathrm{Lu}(1)-\mathrm{C}(4)$ & $129.58(17)$ & $C(2)-C(3)-\operatorname{Lu}(1)$ & $73.4(3)$ \\
\hline $\mathrm{N}(1)-\mathrm{Lu}(1)-\mathrm{C}(4)$ & $81.86(16)$ & $C(8)-C(3)-\operatorname{Lu}(1)$ & $122.3(4)$ \\
\hline $\mathrm{C}(2)-\mathrm{Lu}(1)-\mathrm{C}(4)$ & $51.20(16)$ & $\mathrm{C}(1)-\mathrm{C}(2)-\mathrm{C}(3)$ & $108.1(5)$ \\
\hline $\mathrm{C}(1)-\mathrm{Lu}(1)-\mathrm{C}(4)$ & $50.63(18)$ & $C(1)-C(2)-C(7)$ & $126.1(6)$ \\
\hline $\mathrm{C}(5)-\mathrm{Lu}(1)-\mathrm{C}(4)$ & $30.51(18)$ & $C(3)-C(2)-C(7)$ & $125.5(6)$ \\
\hline $\mathrm{C}(3)-\mathrm{Lu}(1)-\mathrm{C}(4)$ & $31.21(17)$ & $C(1)-C(2)-\operatorname{Lu}(1)$ & $74.7(3)$ \\
\hline $\mathrm{C}(44)-\mathrm{Si}(1)-\mathrm{C}(26)$ & $112.3(10)$ & $C(3)-C(2)-L u(1)$ & $75.1(3)$ \\
\hline $\mathrm{C}(44)-\mathrm{Si}(1)-\mathrm{C}\left(42^{\prime}\right)$ & $88.4(19)$ & $C(7)-C(2)-L u(1)$ & $121.1(4)$ \\
\hline $\mathrm{C}(26)-\mathrm{Si}(1)-\mathrm{C}\left(42^{\prime}\right)$ & 121.1(11) & $C(5)-C(4)-C(3)$ & $108.4(5)$ \\
\hline $\mathrm{C}(44)-\mathrm{Si}(1)-\mathrm{C}(43)$ & $112.3(7)$ & $C(5)-C(4)-C(9)$ & $126.0(6)$ \\
\hline C(26)-Si(1)-C(43) & 113.6(3) & $\mathrm{C}(3)-\mathrm{C}(4)-\mathrm{C}(9)$ & $124.9(6)$ \\
\hline $\mathrm{C}\left(42^{\prime}\right)-\mathrm{Si}(1)-\mathrm{C}(43)$ & $106.6(13)$ & $C(5)-C(4)-L u(1)$ & $74.3(3)$ \\
\hline
\end{tabular}




\begin{tabular}{|c|c|c|c|}
\hline $\mathrm{C}(44)-\mathrm{Si}(1)-\mathrm{C}(42)$ & $106.5(18)$ & $C(3)-C(4)-\operatorname{Lu}(1)$ & $74.3(3)$ \\
\hline $\mathrm{C}(26)-\mathrm{Si}(1)-\mathrm{C}(42)$ & $106.7(10)$ & $\mathrm{C}(9)-\mathrm{C}(4)-\mathrm{Lu}(1)$ & $124.9(4)$ \\
\hline $\mathrm{C}\left(42^{\prime}\right)-\mathrm{Si}(1)-\mathrm{C}(42)$ & $19(2)$ & $\mathrm{C}(30)-\mathrm{C}(27)-\mathrm{C}(28)$ & $110.2(6)$ \\
\hline $\mathrm{C}(43)-\mathrm{Si}(1)-\mathrm{C}(42)$ & $104.8(13)$ & $\mathrm{C}(30)-\mathrm{C}(27)-\mathrm{C}(29)$ & $109.0(6)$ \\
\hline C(44)-Si(1)-C(44') & $21.5(14)$ & $\mathrm{C}(28)-\mathrm{C}(27)-\mathrm{C}(29)$ & $105.9(5)$ \\
\hline$C(26)-\operatorname{Si}(1)-C\left(44^{\prime}\right)$ & $110.6(9)$ & $C(30)-C(27)-C(23)$ & $109.3(5)$ \\
\hline $\mathrm{C}\left(42^{\prime}\right)-\mathrm{Si}(1)-\mathrm{C}\left(44^{\prime}\right)$ & $106.4(18)$ & $\mathrm{C}(28)-\mathrm{C}(27)-\mathrm{C}(23)$ & $112.1(5)$ \\
\hline C(43)-Si(1)-C(44') & $95.2(6)$ & $C(29)-C(27)-C(23)$ & $110.3(4)$ \\
\hline $\mathrm{C}(42)-\mathrm{Si}(1)-\mathrm{C}\left(44^{\prime}\right)$ & $125.4(16)$ & $C(37)-C(35)-C(36)$ & $110.0(11)$ \\
\hline $\mathrm{C}(15)-\mathrm{N}(1)-\mathrm{C}(11)$ & $117.4(4)$ & $\mathrm{C}(37)-\mathrm{C}(35)-\mathrm{C}(38)$ & $101.3(15)$ \\
\hline $\mathrm{C}(15)-\mathrm{N}(1)-\mathrm{Lu}(1)$ & $120.1(3)$ & $\mathrm{C}(36)-\mathrm{C}(35)-\mathrm{C}(38)$ & $107.1(6)$ \\
\hline $\mathrm{C}(11)-\mathrm{N}(1)-\mathrm{Lu}(1)$ & $122.6(3)$ & $\mathrm{C}(37)-\mathrm{C}(35)-\mathrm{C}(18)$ & $114.8(11)$ \\
\hline $\mathrm{C}(20)-\mathrm{N}(2)-\mathrm{C}(16)$ & $114.2(4)$ & $\mathrm{C}(36)-\mathrm{C}(35)-\mathrm{C}(18)$ & $112.5(4)$ \\
\hline $\mathrm{C}(20)-\mathrm{N}(2)-\mathrm{Lu}(1)$ & $117.4(3)$ & $\mathrm{C}(38)-\mathrm{C}(35)-\mathrm{C}(18)$ & $110.4(5)$ \\
\hline $\mathrm{C}(16)-\mathrm{N}(2)-\mathrm{Lu}(1)$ & $125.6(3)$ & $\mathrm{C}(37)-\mathrm{C}(35)-\mathrm{C}\left(37^{\prime}\right)$ & $15(3)$ \\
\hline $\mathrm{C}(25)-\mathrm{N}(3)-\mathrm{C}(21)$ & $117.6(4)$ & $C(36)-C(35)-C\left(37^{\prime}\right)$ & $109.5(16)$ \\
\hline $\mathrm{C}(25)-\mathrm{N}(3)-\mathrm{Lu}(1)$ & $125.3(3)$ & $\mathrm{C}(38)-\mathrm{C}(35)-\mathrm{C}\left(37^{\prime}\right)$ & $114.5(18)$ \\
\hline $\mathrm{C}(21)-\mathrm{N}(3)-\mathrm{Lu}(1)$ & $116.6(3)$ & $\mathrm{C}(18)-\mathrm{C}(35)-\mathrm{C}\left(37^{\prime}\right)$ & $103.1(12)$ \\
\hline $\mathrm{N}(1)-\mathrm{C}(15)-\mathrm{C}(14)$ & $120.9(4)$ & $\mathrm{C}(34)-\mathrm{C}(31)-\mathrm{C}(32)$ & $108.8(5)$ \\
\hline $\mathrm{N}(1)-\mathrm{C}(15)-\mathrm{C}(16)$ & $117.0(4)$ & $\mathrm{C}(34)-\mathrm{C}(31)-\mathrm{C}(13)$ & $112.0(4)$ \\
\hline$C(14)-C(15)-C(16)$ & $122.1(4)$ & $\mathrm{C}(32)-\mathrm{C}(31)-\mathrm{C}(13)$ & $109.6(5)$ \\
\hline $\mathrm{N}(2)-\mathrm{C}(20)-\mathrm{C}(19)$ & $122.3(4)$ & $\mathrm{C}(34)-\mathrm{C}(31)-\mathrm{C}(33)$ & $107.7(6)$ \\
\hline $\mathrm{N}(2)-\mathrm{C}(20)-\mathrm{C}(21)$ & $114.1(4)$ & $\mathrm{C}(32)-\mathrm{C}(31)-\mathrm{C}(33)$ & 111.3(6) \\
\hline$C(19)-C(20)-C(21)$ & $123.0(4)$ & $\mathrm{C}(13)-\mathrm{C}(31)-\mathrm{C}(33)$ & $107.4(5)$ \\
\hline $\mathrm{N}(3)-\mathrm{C}(21)-\mathrm{C}(22)$ & $120.9(4)$ & $\mathrm{C}(37)-\mathrm{C}\left(37^{\prime}\right)-\mathrm{C}(35)$ & $69(9)$ \\
\hline $\mathrm{N}(3)-\mathrm{C}(21)-\mathrm{C}(20)$ & $114.1(4)$ & $\mathrm{C}\left(37^{\prime}\right)-\mathrm{C}(37)-\mathrm{C}(35)$ & $96(8)$ \\
\hline$C(22)-C(21)-C(20)$ & $124.9(4)$ & $\mathrm{C}(41)-\mathrm{Si}(2)-\mathrm{C}(40)$ & $110.4(3)$ \\
\hline $\mathrm{C}(22)-\mathrm{C}(23)-\mathrm{C}(24)$ & $116.3(4)$ & $\mathrm{C}(41)-\mathrm{Si}(2)-\mathrm{C}(45)$ & $106.8(3)$ \\
\hline $\mathrm{C}(22)-\mathrm{C}(23)-\mathrm{C}(27)$ & $123.6(4)$ & $\mathrm{C}(40)-\mathrm{Si}(2)-\mathrm{C}(45)$ & $108.0(3)$ \\
\hline $\mathrm{C}(24)-\mathrm{C}(23)-\mathrm{C}(27)$ & $120.0(4)$ & $\mathrm{C}(41)-\mathrm{Si}(2)-\mathrm{C}(39)$ & $112.6(3)$ \\
\hline $\mathrm{N}(2)-\mathrm{C}(16)-\mathrm{C}(17)$ & $111.0(4)$ & $\mathrm{C}(40)-\mathrm{Si}(2)-\mathrm{C}(39)$ & $112.2(3)$ \\
\hline $\mathrm{N}(2)-\mathrm{C}(16)-\mathrm{C}(15)$ & $108.6(4)$ & $\mathrm{C}(45)-\mathrm{Si}(2)-\mathrm{C}(39)$ & $106.5(3)$ \\
\hline$C(17)-C(16)-C(15)$ & $111.9(4)$ & $\mathrm{C}(16)-\mathrm{C}(39)-\mathrm{Si}(2)$ & $121.7(3)$ \\
\hline $\mathrm{N}(2)-\mathrm{C}(16)-\mathrm{C}(39)$ & $108.5(4)$ & $\mathrm{C}\left(42^{\prime}\right)-\mathrm{C}(42)-\mathrm{Si}(1)$ & $71(7)$ \\
\hline$C(17)-C(16)-C(39)$ & $107.5(4)$ & $\mathrm{C}(42)-\mathrm{C}\left(42^{\prime}\right)-\mathrm{Si}(1)$ & $90(6)$ \\
\hline $\mathrm{C}(15)-\mathrm{C}(16)-\mathrm{C}(39)$ & $109.3(4)$ & $\mathrm{C}\left(44^{\prime}\right)-\mathrm{C}(44)-\mathrm{Si}(1)$ & $94(4)$ \\
\hline $\mathrm{C}(20)-\mathrm{C}(19)-\mathrm{C}(18)$ & $118.6(4)$ & $\mathrm{C}(44)-\mathrm{C}\left(44^{\prime}\right)-\mathrm{Si}(1)$ & $65(4)$ \\
\hline
\end{tabular}




\section{References}

1. Arndt, S.; Voth, P.; Spaniol, T. P.; Okuda, J. Organometallics 2000, 19, 4690-4700.

2. Cameron, T. M.; Gordon, J. C.; Scott, B. L. Organometallics 2004, 23, 2995-3002.

3. Cao, X.; Dolg, M. J. Chem. Phys. 2001, 115, 7348-7355.

4. SMART-NT 4; Bruker AXS, Inc.: Madison, WI 53719, 1996.

5. SAINT-NT 5.050; Bruker AXS, Inc.: Madison, WI 53719, 1998.

6. Sheldrick, G. $S A D A B S$, first release; University of Göttingen: Germany.

7. SHELXTL Version 5.10; Bruker AXS, Inc.: Madison, WI 53719, 1997. 